\title{
Konsensus-Statement - Schlaganfallprophylaxe bei nicht-valvulärem Vorhofflimmern unter besonderer Berücksichtigung der direkten oralen Antikoagulanzien
}

\begin{abstract}
Ingrid Pabinger · Wilfried Lang · Franz Xaver Roithinger · Franz Weidinger · Sabine Eichinger-Hasenauer · Reinhold Glehr · Walter-Michael Halbmayer · Hans-Peter Haring • Bernd Jilma · Hans Christian Korninger • Sibylle Kozek-Langenecker · Paul Kyrle · Herbert Watzke · Ansgar Weltermann · Johann Willeit · Kurt Huber · Österreichische Kardiologische Gesellschaft (ÖKG), Österreichische Gesellschaft für Hämatologie \& Medizinische Onkologie (OeGHO), Österreichische Schlaganfall-Gesellschaft (ÖGSF), Österreichische Gesellschaft für Innere Medizin (ÖGIM), Österreichische Gesellschaft für Anästhesiologie, Reanimation und Intensivmedizin (ÖGARI), Österreichische Gesellschaft für Allgemeinund Familienmedizin (ÖGAM)
\end{abstract}

Eingegangen: 6. Mai 2014 / Angenommen: 9. August 2014 / Online publiziert: 3. Oktober 2014 (c) Die Autor(en) 2014. Dieser Artikel ist auf Springerlink.com mit Open Access verfügbar.

Zusammenfassung Die Einführung neuer direkter oraler Antikoagulanzien hat die Therapie des nichtvalvulären Vorhofflimmerns verändert - diese Veränderungen spiegeln sich jedoch noch nicht vollständig in den aktuellen Leitlinien wider. Das vorliegende Konsensusstatement, an dem sechs österreichische Fachgesellschaften beteiligt sind, gibt aufgrund der vorliegenden Evidenz und der bisher publizierten Leitlinien einen Leitfaden

Univ.-Prof. Dr. I. Pabinger $(\bowtie)$.

Univ.-Prof. Dr. S. Eichinger-Hasenauer - Univ.-Prof. Dr. P. Kyrle

Klin. Abt. für Hämatologie u. Hämostaseologie,

Univ.-Klin. f. Innere Medizin I, MedUni Wien,

Währinger Gürtel 18-20,

1090 Wien, Österreich

E-Mail: ingrid.pabinger@meduniwien.ac.at

Prim. Univ.-Prof. Dr. W. Lang

Neurologische Abt., KH d. Barmh. Brüder,

Wien, Österreich

Prim. Univ.-Doz. Dr. F. X. Roithinger

Interne Abt., LKH Mödling, Mödling, Österreich

Prim. Univ.-Prof. Dr. F. Weidinger

2. Med. Abt., KH Rudolfstiftung,

Wien, Österreich

Dr. R. Glehr

Arzt f. Allgemeinmedizin,

Hartberg, Österreich

Univ.-Doz. Dr. W.-M. Halbmayer

Inst. f. Labormedizin, KH Hietzing,

Wien, Österreich für das aktuelle Vorgehen bei der Thromboembolie-Prophylaxe des nichtvalvulären Vorhofflimmerns. Weiters werden auch spezielle Themen beleuchtet, wie Laborveränderungen und deren Interpretation unter direkten neuen Antikoagulantien, Therapie von Blutungen, Vorgehen bei Operationen und bei Kardioversion und Ablation und spezifische neurologische Aspekte. Ab einem $\mathrm{CHA}_{2} \mathrm{DS}_{2}$-VASc-Score von $\geq 2$ wird eine Antikoagulation

a.o.Univ.-Prof. Dr. B. Jilma

Univ.-Klin. f. Klin. Pharmakologie, MedUni Wien,

Wien, Österreich

Univ.-Doz. Dr. H. C. Korninger

Internist im UKH Lorenz Böhler, Wien, Österreich

Prim. Univ.-Doz. Dr. S. Kozek-Langenecker

Abt. f. Anästhesie u. Intensivmedizin, Ev. KH,

Wien, Österreich

Univ.-Prof. Dr. H. Watzke

Klin. Abt. f. Palliativmedizin, Univ.-Klin. f. Innere Medizin I,

MedUni Wien, Wien, Österreich

Prim. Univ.-Doz. Dr. A. Weltermann

Abt. f. Interne 1, KH d. Elisabethinen,

Linz, Österreich

Univ.-Prof. Dr. J. Willeit

Univ.-Klin. f. Neurologie, MedUni Innsbruck,

Innsbruck, Österreich

Prim. Univ.-Prof. Dr. K. Huber

3. Med. Abt., Wilhelminenspital,

Wien, Österreich 
mit einem hohen Evidenzgrad (1A) empfohlen. Am Schluss des Konsensusstatements werden noch Überlegungen für eine Reihe spezieller Patientengruppen angestellt, um den behandelnden Arzt bei individuellen Patientenentscheidungen zu unterstützen.

Schlüsselwörter Direkte orale Antikoagulanzien · Nichtvalvuläres Vorhofflimmern · Konsensusstatement

\section{Consensus statement: stroke prevention in nonvalvular atrial fibrillation in special consideration of the new direct oral anticoagulants}

Summary The introduction of new direct oral anticoagulants has changed the treatment of nonvalvular atrial fibrillation. However, these changes are not yet fully reflected in current guidelines.This consensus statement, endorsed by six Austrian medical societies, provides guidance to current prophylacticapproaches of thromboembolic events in nonvalvular atrial fibrillation on the basis of current evidence and published guidelines.

Furthermore, some special subjects are treated, like changes in laboratory parameters and their interpretation under treatment with direct oral anticoagulants, treatment of bleedings, approach to operations, cardioversion and ablation, and specific neurological aspects. For a $\mathrm{CHA}_{2} \mathrm{DS}_{2}$-VASc-Score of $\geq 2$, anticoagulation is recommended with a high level of evidence (1A). At the end of the consensus statement, recommendations for a number of specific patient subgroups can be found, in order to help treating physicians to arrive at appropriate therapeutic decisions.

Keywords Direct oral anticoagulants · Nonvalvular atrial fibrillation - Consensusstatement

\section{Einleitung}

Die Schlaganfall-Prophylaxe bei Patienten mit Vorhofflimmern (VHF - zu den im Text verwendeten Abkürzungen siehe auch Tab. 1) ist einerseits aufgrund der Häufigkeit von VHF, andererseits wegen der Entwicklung neuer pharmakologischer Möglichkeiten der oralen Antikoagulation Gegenstand einer Reihe neuer internationaler Leitlinien geworden. Vor allem seien hier die aktuellen Guidelines der Europäischen Kardiologischen Gesellschaft (ESC) [10, 11] sowie des „American College of Chest Physicians“ (ACCP) [68] genannt, die beide 2012 in aktuellen Fassungen erschienen sind.

Die Zahl der VHF-Patienten, die tatsächlich eine antithrombotische Therapie erhalten, ist wesentlich geringer als die Zahl jener VHF-Patienten, bei denen eine solche Therapie indiziert wäre. Dies zeigen z. B. Daten aus einem kanadischen Register. Hier waren VHF-Patienten, die mit einem ischämischen Schlaganfall aufgenommen wurden, nur zu $10 \%$ im therapeutischen Bereich mit
Tab. 1 Verwendete Abkürzungen

\begin{tabular}{|c|c|}
\hline ACCP & American College of Chest Physicians \\
\hline AKS & Akutes Koronarsyndrom \\
\hline $\mathrm{aPTT}$ & Aktivierte partielle Thromboplastinzeit \\
\hline ASS & Azetylsalizylsäure \\
\hline BMS & Bare-Metal Stent \\
\hline $\begin{array}{l}\mathrm{CHA}_{2} \mathrm{DS}_{2}- \\
\text { VASc-Score }\end{array}$ & $\begin{array}{l}\text { Risikoscore, der sich aus den Parametern Herzinsuffizienz } \\
\text { (Congestive Heart Failure), Hypertonie (je } 1 \text { Punkt), Alter } \\
\text { > 75a (2 Punkte), Diabetes (1 Punkt), Schlaganfall (2 Punkte), } \\
\text { arterielle Gefäßerkrankungen (Vascular Disease - } 1 \text { Punkt), } \\
\text { Alter 65-74a (1 Punkt) und Geschlecht (Sex category - 1 } \\
\text { Punkt für weiblich) zusammensetzt (s. auch Tab. 7) }\end{array}$ \\
\hline $\mathrm{Cl}$ & Konfidenzintervall \\
\hline CYP & Cytochrom-P-450 \\
\hline DES & Drug-Eluting Stent \\
\hline DOAK & $\begin{array}{l}\text { Direct Oral Anticoagulant (oft auch als „NOAK“ = „Novel Oral } \\
\text { Anticoagulant" bezeichnet) }\end{array}$ \\
\hline DWI & Diffusion-Weighted Imaging (im MRT) \\
\hline ESC & European Society of Cardiology \\
\hline FFP & Fresh-Frozen Plasma \\
\hline$H R$ & Hazard Ratio \\
\hline HWZ & Halbwertszeit \\
\hline INR & International Normalized Ratio \\
\hline ITT & Intention to Treat \\
\hline KHK & Koronare Herzkrankheit \\
\hline $\mathrm{KrCl}$ & Kreatinin-Clearance \\
\hline MRT & Magnetresonanztomographie \\
\hline $\mathrm{NI}$ & Niereninsuffizienz \\
\hline NIHSS & National Institute of Health Stroke Scale \\
\hline NSAR & Nichtsteroidale Antirheumatika \\
\hline NUL & Nichtunterlegenheit \\
\hline OAK & $\begin{array}{l}\text { Orale Antikoagulation (hier sind, sofern nicht genauer an- } \\
\text { gegeben, alle Möglichkeiten der OAK gemeint, also sowohl } \\
\text { VKA als auch DOAK) }\end{array}$ \\
\hline PCC & Prothrombinkomplex-Konzentrat \\
\hline P-gp & P-Glykoproteintransporter in der Darmwand \\
\hline PTZ & Prothrombinzeit, Thromboplastinzeit \\
\hline $\mathrm{RCT}$ & Randomisierte kontrollierte Studie \\
\hline rFVlla & Rekombinanter aktivierter Faktor VII \\
\hline $\mathrm{RR}$ & Relatives Risiko \\
\hline TEE & Transösophageale Echokardiographie \\
\hline TIA & Transitorisch-Ischämische Attacke \\
\hline TTR & Time in Therapeutic Range (bezieht sich auf die INR bei VKA) \\
\hline ÜL & Überlegenheit \\
\hline VHF & Vorhofflimmern \\
\hline VKA & Vitamin-K-Antagonist \\
\hline
\end{tabular}

VKA antikoaguliert, weitere $29 \%$ im subtherapeutischen Bereich; weitere $31 \%$ hatten Plättchenhemmer erhalten, $29 \%$ erhielten überhaupt keine antithrombotische Therapie [28]. Es ist zu hoffen, dass sich diese Situation mit der Einführung der „Direkten oralen Antikoagulanzien“ (DOAK) bessern und damit auch in Österreich die Zahl der vermeidbaren Insulte reduzieren wird. Über natio- 
konsensus bericht

Tab. 2 Pharmakologie der DOAK. (Quellen: [2, 3, 4, 9, 27, 50])

\begin{tabular}{|c|c|c|c|c|}
\hline & Dabigatran & Rivaroxaban & Apixaban & Edoxaban \\
\hline Wirkmechanismus & Direkte Hemmung von Thrombin & $\begin{array}{l}\text { Direkte Hemmung von Faktor } \\
\text { Xa }\end{array}$ & $\begin{array}{l}\text { Direkte Hemmung von Faktor } \\
\mathrm{Xa}\end{array}$ & $\begin{array}{l}\text { Direkte Hemmung von Faktor } \\
\mathrm{Xa}\end{array}$ \\
\hline Bioverfügbarkeit & $6,5 \%$ & $80-100 \%$ & $50 \%$ & $62 \%$ \\
\hline Applikationsweg & Oral & Oral & Oral & Oral \\
\hline Pro-Drug & $\mathrm{Ja}$ & Nein & Nein & Nein \\
\hline $\begin{array}{l}\text { Interaktion mit } \\
\text { Nahrungsmitteln }\end{array}$ & Nein & Nein & Nein & Nein \\
\hline Renale Ausscheidung & $85 \%$ & $33 \%$ & $27 \%$ & $37-50 \%$ \\
\hline Mediane HWZa,b & $12-14 \mathrm{~h}$ & $\begin{array}{l}7-11 \mathrm{~h}(11-13 \mathrm{~h} \text { bei älteren } \\
\text { Patienten) }\end{array}$ & $12 \mathrm{~h}$ & $6-11$ \\
\hline$T_{\max }$ & $0,5-2 \mathrm{~h}$ & $2-4 \mathrm{~h}$ & $3-4 \mathrm{~h}$ & $1-2 \mathrm{~h}$ \\
\hline Metabolisierung & $\begin{array}{l}\text { Nicht über CYP; Hauptteil unverändert } \\
\text { renal ausgeschieden; Glukuronidie- } \\
\text { rung; Substrat von P-gp }\end{array}$ & $\begin{array}{l}\text { CYP3A4, CYP2J2 sowie CYP- } \\
\text { unabhängige Mechanismen; } \\
\text { Substrat von P-gp }\end{array}$ & $\begin{array}{l}\text { V. a. CYP3A4/5, in geringerem } \\
\text { Umfang CYP1A2, 2C8, 2C9, } \\
\text { 2C19 und 2J2; Substrat von } \\
\text { P-gp }\end{array}$ & V. a. CYP3A4 \\
\hline Interaktionen ${ }^{c}$ & $\begin{array}{l}\text { Bei gleichzeitiger Anwendung } \\
\text { von starken P-gp-Hemmern (z. B. } \\
\text { Amiodaron, Verapamil, Chinidin, Ke- } \\
\text { toconazol und Clarithromycin) ist eine } \\
\text { erhöhte Dabigatran-Plasmakonzen- } \\
\text { tration zu erwarten. Bei gleichzeitiger } \\
\text { Anwendung von P-gp-Induktoren } \\
\text { (wie Rifampicin, Johanniskraut, } \\
\text { Carbamazepin oder Phenytoin) ist } \\
\text { ein verringerter Dabigatran-Plasma- } \\
\text { spiegel zu erwarten }\end{array}$ & $\begin{array}{l}\text { Inhibitoren von CYP3A4 } \\
\text { und P-gp (AUC und Cmax v. } \\
\text { Rivaroxaban } \uparrow \text { ), z. B. Azol- } \\
\text { Antimykotika, HIV-Protease- } \\
\text { inhibitoren Induktoren von } \\
\text { CYP3A4 und P-gp (AUC und } \\
\text { Cmax v. Rivaroxaban } \downarrow \text { ), wie } \\
\text { z. B. Rifampicin, Phenytoin, } \\
\text { Carbamazepin, Phenobarbital, } \\
\text { Johanniskraut }\end{array}$ & $\begin{array}{l}\text { Inhibitoren von CYP3A4 und } \\
\text { P-gp (AUC und C }{ }_{\text {max }} \text { v. Apixa- } \\
\text { ban } \uparrow \text { ), z. B. Azol-Antimyko- } \\
\text { tika, HIV-Proteaseinhibitoren } \\
\text { Induktoren v. CYP3A4 und } \\
\text { P-gp (AUC und C }{ }_{\text {max }} \text { v. Apixa- } \\
\text { ban } \downarrow \text { ), wie z. B. Rifampicin, } \\
\text { Phenytoin, Carbamazepin, } \\
\text { Phenobarbital, Johanniskraut }\end{array}$ & $\begin{array}{l}\text { Substrat für P-gp; Dosis- } \\
\text { reduktion kann notwendig } \\
\text { werden, wenn gleichzeitig } \\
\text { starke P-gp-Inhibitoren } \\
\text { (z. B. Verapamil, Chinidin, } \\
\text { Amiodaron oder Droneda- } \\
\text { ron) verabreicht werden; } \\
\text { gleichzeitige Verabreichung } \\
\text { von NSAR kann Blutungszeit } \\
\text { verlängern }\end{array}$ \\
\hline \multicolumn{5}{|c|}{$\begin{array}{l}\text { aZu den verwendeten Abkürzungen siehe Tab. } 1 \\
\text { bFür alle DOAK gilt, dass beim individuellen Patienten die Halbwertszeiten wesentlich stärker schwanken können, als hier in der Tabelle angegeben, z. B. bis } \\
\text { über } 20 \text { h, was - abgesehen von der Nierenfunktion - klinisch von Bedeutung sein kann } \\
\text { cFür alle hier erwähnten Substanzen gilt, dass die gleichzeitige Verabreichung anderer, die Blutungsneigung erhöhender Substanzen, das Blutungsrisiko erhöhen } \\
\text { kann. Für eine detaillierte Darstellung von Interaktionen zwischen DOAK und anderen Medikamenten siehe [34] }\end{array}$} \\
\hline
\end{tabular}

nale Schlaganfall-Register könnte eine solche Veränderung in Österreich laufend überprüft werden.

Bevor eine endgültige Entscheidung über den Stellenwert der DOAK getroffen wird, sollten allerdings auch die Daten aus weiteren prospektiven Studien, aber vor allem auch aus derzeit laufenden großen Registern abgewartet werden, um ein vollständigeres und realistischeres Bild mit Nähe zur klinischen Routine zu erhalten.

Das vorliegende Konsensus-Statement wird von sechs Fachgesellschaften getragen. Außerdem wirkten Vertreter einer Reihe weiterer Fachdisziplinen, wie Anästhesiologie, Klinische Pharmakologie, Labormedizin sowie Allgemeinmedizin, am Zustandekommen des vorliegenden Konsensus-Statements mit, dessen Ziel es nicht ist, die vorliegende Evidenz neu zu bewerten, sondern vielmehr die aktuelle Datenlage für die klinische Praxis in Österreich darzustellen.

\section{Direkte orale Antikoagulanzien (DOAK)}

Als direkte orale Antikoagulanzien - DOAK - werden der direkte Thrombininhibitor Dabigatran sowie die direkten Faktor-Xa-Inhibitoren Apixaban, Rivaroxaban und Edoxaban bezeichnet. Tabelle 2 listet wichtige pharmakologische Eckdaten der DOAK auf.
Die Phase-III-Studien zu den einzelnen DOAK sind aufgrund des Fehlens von „head-to-head“-Studien und wegen der Unterschiede in den untersuchten Patientenkollektiven nicht direkt vergleichbar. Daher sind auch Metaanalysen, die das dennoch versuchen, mit Vorsicht zu interpretieren. Unterschiede zwischen den einzelnen Substanzen sind nur bedingt darstellbar, was auch bei den folgenden Tab. 2 und $3 \mathrm{zu}$ beachten ist.

Dabigatran wurde in der RE-LY-Studie in zwei randomisierten, verblindeten Dosierungen (110 und $150 \mathrm{mg}$ ) mit dem VKA Warfarin (INR 2,0-3,0) verglichen [13]. Diese sehr groß angelegte Studie im Nichtunterlegenheits-Design umfasste 18.113 Patienten mit VHF und mindestens einem zusätzlichen Risikofaktor. Dabei war der Warfarin-Arm unverblindet, lediglich die beiden Dabigatran-Arme waren gegeneinander verblindet. Die mittlere Beobachtungsdauer betrug zwei Jahre. Der primäre Wirksamkeitsendpunkt war Schlaganfall oder systemische Embolie, der primäre Sicherheitsendpunkt schwere Blutung.

Der primäre Wirksamkeitsendpunkt trat bei $1,69 \%$ pro Jahr in der Warfarin-Gruppe, bei 1,53\%/a unter Dabigatran $2 \times 110 \mathrm{mg}(p<0,001$ für Nichtunterlegenheit) und bei $1,11 \%$ /a unter Dabigatran $2 \times 150 \mathrm{mg}$ auf $(p<0,001$ für Überlegenheit). Die Rate schwerer Blutungen lag unter Warfarin bei $3,36 \%$ pro Jahr, unter $2 \times 110 \mathrm{mg}$ Dabigatran 


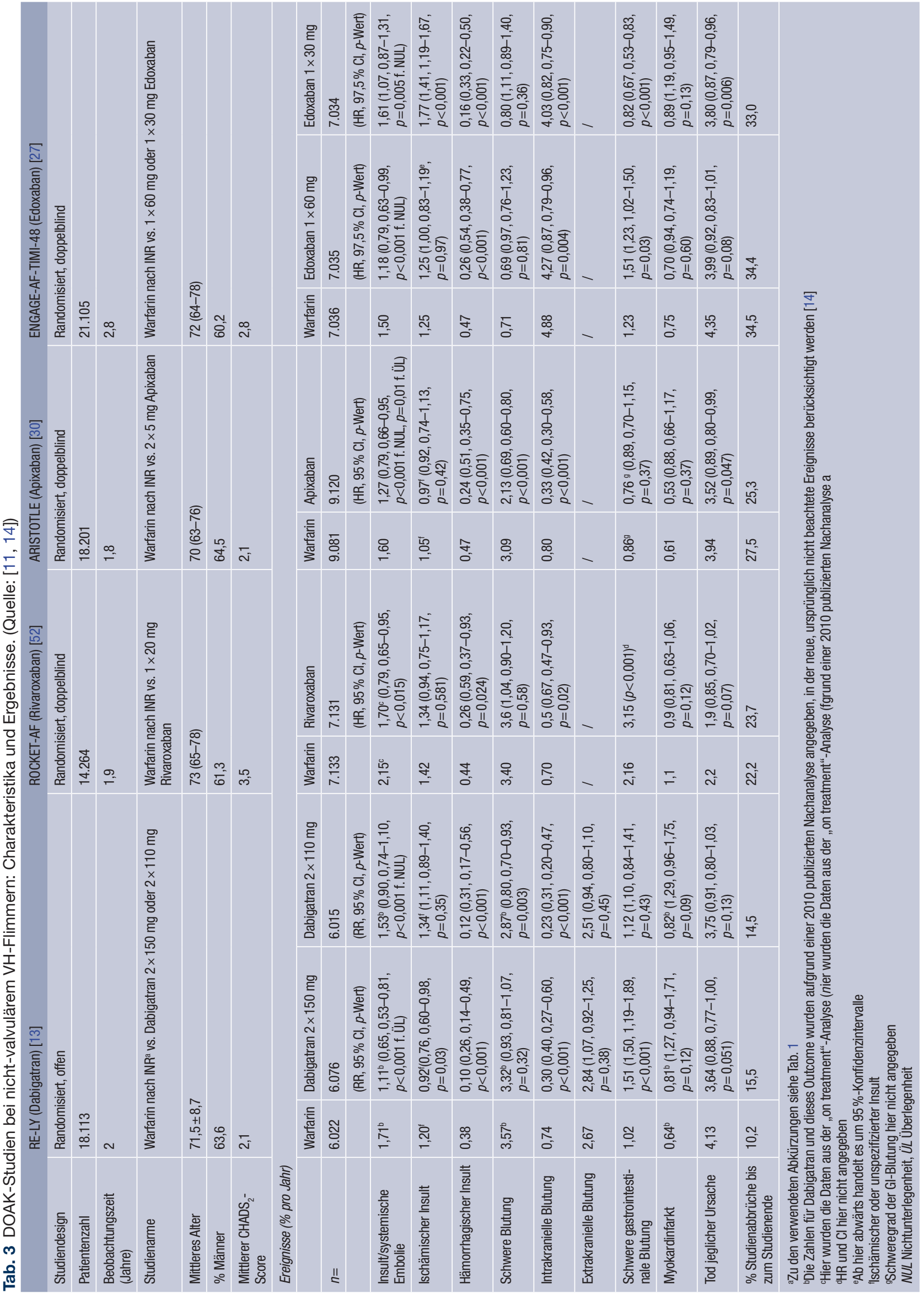


bei $2,71 \% / \mathrm{a}(p=0,003)$ und unter $2 \times 150 \mathrm{mg}$ Dabigatran bei $3,11 \% / \mathrm{a}(p=0,31)$. Unter $2 \times 150 \mathrm{mg}$ Dabigatran waren die Raten für hämorrhagischen Insult, ischämischen oder nicht-spezifizierten Insult sowie Insult ohne und mit Behinderung jeweils signifikant niedriger als unter Warfarin.

Allerdings traten unter $2 \times 150 \mathrm{mg}$ Dabigatran signifikant mehr gastrointestinale Blutungen auf als unter Warfarin [14]. Weiters traten unter $2 \times 150 \mathrm{mg}$ Dabigatran numerisch mehr Myokardinfarkte auf, wobei allerdings in einer revidierten Analyse der RE-LY-Studie dieses Ergebnis statistisch nicht mehr signifikant war [14]. Die kardiovaskuläre Mortalität wurde dadurch jedoch nicht beeinflusst.

In der RE-LY-Extension-Studie („RELY-ABLE“) konnte während eines im Mittel 2,3 Jahre dauernden Follow-up eine höhere Rate an schweren Blutungen unter der $2 \times 150$ mg-Dosierung gegenüber der $2 \times 110 \mathrm{mg}$-Dosierung gezeigt werden, während die Raten für Schlaganfall und Mortalität für beide Dosierungen vergleichbar waren [15]. Insgesamt kann aber von einer sehr guten LangzeitEffektivität und -Sicherheit ausgegangen werden.

Die Tatsache, dass spontane Berichte über Blutungen unter Dabigatran erheblich häufiger abgegeben wurden als unter VKA, führte zu einer unabhängigen Untersuchung der US-Zulassungsbehörde FDA. Diese kam zu dem Schluss, dass es sich hier weitestgehend um einen „Reporting Bias“ aufgrund der Neuheit des Produkts und nicht um eine tatsächlich erhöhte Blutungsrate handelt [58].

Aufgrund der längeren Verfügbarkeit von Dabigatran im Vergleich zu den anderen am Markt befindlichen DOAK sind die klinischen Erfahrungen größer und manche Problemstellungen besser einzuschätzen und zu managen [38].

Rivaroxaban (20 mg pro Tag; $15 \mathrm{mg} / \mathrm{d}$ bei $\mathrm{KrCl}$ 30-49 $\mathrm{ml} / \mathrm{min}$ ) wurde bei 14.264 Patienten mit nichtvalvulärem VHF in der ROCKET-AF-Studie, ebenfalls im Vergleich zu Warfarin (INR 2,0-3,0), untersucht [52]. Die Patienten mussten Insult-Risikofaktoren oder einen Insult bzw. eine TIA in der Anamnese aufweisen. Auch hier war der primäre Endpunkt Insult oder systemische Embolie und auch diese Studie war im Nichtunterlegenheits-Design angelegt. Die Nichtunterlegenheitskriterien für Rivaroxaban wurden erfüllt $(p<0,001)$, eine Überlegenheit gegenüber dem VKA konnte in der ITT-Analyse nicht nachgewiesen werden $(p=0,12)$. Der Unterschied zwischen den Ergebnissen der ITT-Population und der On-Treatment-Analyse in dieser Studie ist Gegenstand anhaltender Diskussionen. Eine der Ursachen könnte eine hohe Zahl an vaskulären Ereignissen 2 bis 30 Tage nach Beendigung der Studienmedikation sein - eine Phase, in der viele Patienten keine suffiziente gerinnungshemmende Therapie erhielten. Bei der Gesamtblutungsrate fand sich kein signifikanter Unterschied; unter Rivaroxaban traten jedoch signifikant weniger intrakranielle und tödliche Blutungen auf als unter Warfarin. Hingegen waren die gastrointestinale Blutungsrate unter Rivaroxaban sowie die Rate an Hämoglobinab- fällen um $\geq 2 \mathrm{~g} / \mathrm{dl}$ und an Bluttransfusionen signifikant höher als unter Warfarin [52]. Die INR-Einstellung war in ROCKET-AF generell etwas schlechter als in den anderen Studien, was allerdings in erster Linie durch das Studiendesign erklärbar ist.

Apixaban $(2 \times 5 \mathrm{mg} / \mathrm{d} ; 2 \times 2,5 \mathrm{mg}$, wenn zwei oder mehr der folgenden Kriterien bestanden: Alter $\geq 80$ Jahre, Körpergewicht $\leq 60 \mathrm{~kg}$, Serum-Kreatinin $\geq 1,5 \mathrm{mg} / \mathrm{dl}$ ) wurde in der ARISTOTLE-Studie mit Warfarin (INR 2,0-3,0) verglichen [30]. Teilnehmer waren 18.201 Patienten mit VHF und zumindest einem zusätzlichen Schlaganfall-Risikofaktor. Der primäre Endpunkt war ischämischer oder hämorrhagischer Insult oder systemische Embolie. Die mediane Beobachtungszeit betrug 1,8 Jahre. Der primäre Endpunkt trat unter Apixaban bei 1,27\%/a auf, unter Warfarin bei $1,60 \% / \mathrm{a}(p<0,001$ für Nichtunterlegenheit, $p=0,01$ für Überlegenheit). Die Rate schwerer Blutungen betrug unter Apixaban 2,13\%/a, unter Warfarin 3,09\%/a $(p<0,001)$. Die Gesamtmortalität lag unter Apixaban bei $3,52 \% / a$, unter Warfarin bei 3,94\%/a $(p=0,047)$. Apixaban senkte außerdem die Rate hämorrhagischer Insulte gegenüber Warfarin signifikant, nicht jedoch die Rate ischämischer oder unspezifizierter Schlaganfälle.

Edoxaban wurde in der ENGAGE-AF-TIMI-48-Studie bei mehr als 21.105 Patienten mit dem VKA Warfarin verglichen [27]. Es handelte sich um eine randomisierte, doppelblinde Doppel-Dummy-Studie bei Patienten mit VHF der mittleren bis hohen Risikokategorie. Die Beobachtungszeit lag im Median bei 2,8 Jahren. Der primäre Wirksamkeitsendpunkt war das Eintreten eines Schlaganfalls oder einer systemischen Embolie. Der primäre Sicherheitsendpunkt waren große Blutungen. Die Patienten wurden 1:1:1 randomisiert und erhielten entweder Warfarin, titriert auf eine INR zwischen 2,0 und 3,0 oder $60 \mathrm{mg}$ Edoxaban ("Hochdosis“) oder 30 mg Edoxaban („Niedrigdosis“), jeweils einmal täglich. Patienten mit erhöhtem Blutungsrisiko (ältere Patienten, eingeschränkte Nierenfunktion) erhielten jeweils $50 \%$ der jeweiligen Dosis.

Die annualisierte Rate des primären Endpunkts lag unter Warfarin bei $1,50 \%$, unter Hochdosis-Edoxaban bei $1,18 \%$, unter Niedrigdosis-Edoxaban bei 1,61. Beide Edoxaban-Dosierungen erfüllten die Kriterien für Nichtunterlegenheit gegenüber Warfarin. Die $60 \mathrm{mg}$-Dosierung war in der „on-treatment"-Analyse statistisch dem Warfarin überlegen.

Die annualisierte Rate großer Blutungen betrug unter Warfarin 3,43\%, unter Hochdosis-Edoxaban 2,75\% (HR $0,80 ; p<0,001)$ und unter Niedrigdosis-Edoxaban 1,61\% (HR 0,$47 ; p<0,001$ ). Die annualisierten Raten kardiovaskulärer Todesfälle lagen unter Warfarin bei 3,17\%, unter Hochdosis-Edoxaban bei 2,74\% (HR 0,86; $p=0,01$ ) und unter Niedrigdosis-Edoxaban bei 2,71\% (HR 0,85; $p=0,008$ ). Auch unter Hochdosis-Edoxaban war die Rate gastrointestinaler Blutungen höher als unter VKA, unter Niedrigdosis-Edoxaban jedoch niedriger als unter VKA.

Da zum Zeitpunkt der Schlussredaktion dieses Konsensus-Statements in der EU noch keine Zulassung für Edoxaban in der Indikation VHF vorlag, wird die Substanz 
zwar bereits in den Tab. 2 und 3 berücksichtigt, es wird aber im weiteren Text nicht näher darauf eingegangen.

Zusammenfassend kann gesagt werden, dass hinsichtlich des Outcomes Insult bzw. systemische Embolie jedes der beschriebenen DOAK in Abhängigkeit der statistischen Berechnung gleich gut oder besser wirkt als Warfarin.

Die Rate schwerer Blutungen ist unter Dabigatran $2 \times 110 \mathrm{mg}$, unter Apixaban sowie unter Edoxaban $1 \times 60 \mathrm{mg}$ und $1 \times 30 \mathrm{mg}$ geringer als unter VKA, unter Dabigatran $2 \times 150 \mathrm{mg}$ und unter Rivaroxaban gleich wie unter VKA.

Hinsichtlich der Verhinderung hämorrhagischer Schlaganfälle und intrakranieller Blutungen (d. h. subdurale Blutungen, subarachnoidale Blutungen und primär intrazerebrale Blutungen) ist jede der vier Substanzen signifikant besser als VKA. Hinsichtlich der Verhinderung ischämischer Schlaganfälle zeigte nur Dabigatran $2 \times 150 \mathrm{mg}$ eine signifikant bessere Wirkung gegenüber VKA, während die anderen Substanzen dem VKA gleichwertig waren. (Eine Ausnahme stellt hier Edoxaban $1 \times 30 \mathrm{mg}$ dar. Unter dieser Dosis traten signifikant mehr ischämische Insulte auf als unter Warfarin.)

Tabelle 3 fasst Detail-Outcomes der Schlüsselstudien RE-LY, ROCKET-AF, ARISTOTLE und ENGAGE-AFTIMI-48 zusammen.

\section{Medikamenteninteraktionen mit DOAK}

Einen Überblick der pharmakologischen Kenndaten und auch der wichtigsten Medikamenteninteraktionen der DOAK gibt Tab. 2. Diese Interaktionen kommen über mehrere Mechanismen zustande. Hier ist einerseits der Effluxtransporter in der Darmwand P-gp zu nennen, dessen Substrate die DOAK sind, andererseits das Cytochrom-P450-Enzymsystem, durch welches Rivaroxaban, Apixaban und Edoxaban, nicht jedoch Dabigatran, abgebaut werden.

Konkret sind für Dabigatran Inhibitoren von P-gp wie Ketoconazol, Itraconazol, Cyclosporin und Tacrolimus kontraindiziert. Nicht empfohlen wird die gleichzeitige Gabe von P-gp-Induktoren wie Rifampicin, Johanniskraut, Phenytoin und Carbamazepin, von Proteasehemmern wie z. B. Ritonavir und von Dronedaron. Bei gleichzeitiger Gabe von Verapamil wird eine Dosisreduktion auf $2 \times 110 \mathrm{mg}$ empfohlen. Die Gabe von Pantoprazol reduziert zwar die AUC von Dabigatran um ca. $30 \%$, aber die Ergebnisse einer Subanalyse zeigen, dass es nicht zu einer Abnahme der klinischen Wirksamkeit von Dabigatran kommt. ASS, NSAR und Clopidogrel erhöhen, ebenso wie SSRI, das Blutungsrisiko bei gleichzeitiger Gabe mit Dabigatran. Prasugrel und Ticagrelor sind diesbezüglich nicht untersucht, was auch für die anderen DOAK gilt [3].

Für Rivaroxaban und Apixaban gilt, dass Substanzen, die gleichzeitig P-gp und CYP3A4 hemmen, nicht empfohlen werden. Dies sind vor allem Azol-Antimykotika und HIV-Proteasehemmer. Induktoren von P-gp und
CYP3A4 wie Rifampicin, Carbamazepin, Phenytoin oder Johanniskraut, sollten mit Vorsicht angewandt werden, weil die Plasmaspiegel der DOAK signifikant reduziert werden könnten.

Die Erhöhung des Blutungsrisikos durch Thrombozytenfunktionshemmer und SSRI beruht auf einer pharmakodynamischen Wechselwirkung und gilt für alle DOAK $[2,4]$.

\section{Labor und DOAK}

Die DOAK sind direkte Thrombin- oder Faktor-Xa-Hemmer und werden entsprechend ihrer Zulassung nicht nach einem im Labor bestimmten Gerinnungswert, sondern nach klinischer Indikation und Zulassung dosiert. Ein Labor-Monitoring im herkömmlichen Sinn ist nicht erforderlich. Nierenfunktionstests sind jedoch vor Verordnung und später je nach klinischer Situation wegen Kumulationsgefahr durchzuführen.

Dosisanpassungen der DOAK bei eingeschränkter Nierenfunktion sind daher erforderlich, siehe Tab. 9 (Empfehlungen der Autoren).

In vitro beeinflussen sowohl Thrombin- als auch Faktor-Xa-Hemmer zeitabhängig und in Abhängigkeit vom jeweils verwendeten Reagens zahlreiche Gerinnungstests (Globaltests, Gerinnungsfaktoren und Spezialtests) und können pathologische Gerinnungssituationen vortäuschen (Tab. 4) [31, 48, 63].

Weitere Informationen zu diesem Punkt sind unter [1] zu beziehen.

Die Globalteste PTZ/INR und aPTT sind nicht ausreichend geeignet, klinisch relevante Aussagen zu ermöglichen, und können nur für orientierende Rückschlüsse (relative Intensität) herangezogen werden $[5,6]$. Apixaban beeinflusst die PTZ sehr wenig, sodass keine orientierenden Rückschlüsse zulässig sind [26].

Derzeit können aus den verschiedenen Routine-Gerinnungstests für die verschiedenen DOAK nur wenige klinische Schlussfolgerungen gezogen werden:

- Trotz unterschiedlicher Verfahren der ThrombinzeitMessung lässt eine normale, nicht verlängerte Thrombinzeit (oder auch ECT [Ecarin-Clotting-Time]) einen klinisch relevanten Dabigatranspiegel nahezu ausschließen [5, 6, 59].

- Faktor-Xa-Hemmer scheinen die PTZ (in Abhängigkeit vom verwendeten Reagens) mehr zu beeinflussen als die aPTT. Dabigatran verhält sich offenbar umgekehrt (die aPTT stärker verlängernd als die PTZ), beeinflusst jedoch am stärksten die Thrombinzeit-Messung und damit auch die funktionelle Fibrinogenmessung nach Clauss (Tab. 4) [5, 6, 31].

Im speziellen und auch in manchen Situationen des klinischen Alltags kann die Messung der Wirkung der DOAK sinnvoll sein.

Solche Situationen können u. a. sein: Blutungszwischenfälle, akute operative Eingriffe oder auch Compli- 
Tab. 4 Interferenz von Dabigatran und Rivaroxaban mit Global-Gerinnungsbefunden. (Quelle: Modifiziert nach [36])

\begin{tabular}{|l|l|}
\hline Parameter & $\begin{array}{l}\text { Veränderung (dosisab- } \\
\text { hängig) }\end{array}$ \\
\hline Dabigatran & $\downarrow$ \\
\hline PTZ (\%) & $\uparrow$ \\
\hline INR (Ratio) & $\uparrow \uparrow$ \\
\hline aPTT (sec.) & $\uparrow \uparrow \uparrow$ bis Unmessbarkeit \\
\hline Thrombinzeit (sec.) & $\downarrow \downarrow$ \\
\hline Fibrinogen (mg/dl oder g/l; Clauss, mit <50 U & \\
\hline Thrombin im Reagens) & \\
\hline Rivaroxaban & $\downarrow \downarrow$ \\
\hline PTZ (\%) & $\uparrow \uparrow$ \\
\hline INR (Ratio) & $\uparrow$ \\
\hline aPTT (sec.) & $\leftrightarrow$ \\
\hline Thrombinzeit (sec.) & $\leftrightarrow$ \\
\hline Fibrinogen (mg/dl oder g/l; Clauss) & \\
\hline
\end{tabular}

ance-Überprüfung bei thromboembolischen Ereignissen unter DOAK $[5,6]$.

In einer Reihe österreichischer Spitäler stehen für solche Situationen CE-zugelassene, funktionelle Testsysteme zur Verfügung, für Dabigatran v. a. die verdünnte modifizierte Thrombinzeit (Hemoclot ${ }^{\mathrm{Tm}}$-Test) und für Rivaroxaban chromogene Anti-Xa-Tests (für Apixaban soll in Kürze ein analoges Testsystem verfügbar sein). Auch wenn diese Tests mit der Zielsubstanz kalibriert werden, sind sie auch für andere Antikoagulanzien wie z. B. Hirudin, Argatroban (Hemoclot ${ }^{\mathrm{Tm}}$-Test) oder Heparin, Fondaparinux bzw. andere Xabane (Substanzen mit dem gleichen Wirkmechanismus wie Apixaban oder Rivaroxaban - Anti-Xa-Test) sensitiv - d. h. diese Substanzen werden bei der funktionellen Messung miterfasst.

\section{Akute Situationen: Antikoagulanzien-Reversion, Therapie von Blutungen}

Die Eliminations-HWZ von DOAK (Tab. 2) sind zwar deutlich kürzer als jene von VKA (v. a. jene des in Österreich häufig verwendeten Phenprocoumon, das eine HWZ von $150 \mathrm{~h}$ aufweist, während Acenocoumarol nur eine HWZ von 20h hat), dennoch wird das bloße Absetzen des DOAK nicht in allen Situationen genügen, um eine adäquate Gerinnungssituation zu erreichen.

Spezifische Antidote für die einzelnen DOAK stehen derzeit für die klinische Anwendung noch nicht zur Verfügung. Auf dem Kongress der „American Heart Association“ 2013 wurden allerdings zwei interessante Substanzen vorgestellt, einerseits ein Dabigatran-spezifisches Antidot mit guter Wirksamkeit bei gesunden Probanden [29], andererseits eine Substanz, die imstande zu sein scheint, die DOAK-induzierte Antikoagulation ohne Substanzspezifität aufzuheben [7].

In der Akutsituation können PCC (50IE/kg KG) oder rekombinanter aktivierter Faktor VII (rFVIIa, $90 \mu \mathrm{g} / \mathrm{kg}$ ) in
Tab. 5 Hämodialyse bei Blutungen unter Dabigatran

\begin{tabular}{|c|c|}
\hline \multirow[t]{2}{*}{$\begin{array}{l}\text { Verfahren, } \\
\text { Dauer }\end{array}$} & $\begin{array}{l}\text { Intermittierend: Hämodialyse (HD) oder Hämodiafiltration } \\
\text { (HDF) }\end{array}$ \\
\hline & $\begin{array}{l}\text { Kontinuierlich: (bei instabilen Patienten) jeweils 3-4 h HD, } \\
\text { HDF }\end{array}$ \\
\hline Zugang & Hämofiltrationskatheter \\
\hline \multirow[t]{2}{*}{$\begin{array}{l}\text { Dialyse- } \\
\text { membran }\end{array}$} & $\begin{array}{l}\text { Bei HD: Mid-Flux- oder High-Flux-Membran (zu Low-Flux- } \\
\text { Membranen liegen dzt. keine Daten vor) }\end{array}$ \\
\hline & Bei HDF: High-Flux-Membran \\
\hline $\begin{array}{l}\text { Antikoagu- } \\
\text { lation }\end{array}$ & Zitrat-Antikoagulation \\
\hline Blutfluss & $250-300 \mathrm{ml} / \mathrm{min}$ \\
\hline Dialysatfluss & $500-800 \mathrm{ml} / \mathrm{min}$ \\
\hline $\begin{array}{l}\text { Beurteilung } \\
\text { der Wirkung }\end{array}$ & Thrombinzeit oder Hemoclot ${ }^{\top M}$ \\
\hline
\end{tabular}

Erwägung gezogen werden. Klinische Daten bei Patienten dazu fehlen allerdings weitgehend, jedoch gibt es Daten für die Wirkung auf bestimmte Gerinnungstests bei Probanden [23]. In dieser Studie wurde eine Wirkung von PCC im Sinne einer zumindest teilweisen Aufhebung der Wirkung von Rivaroxaban gezeigt.

Von den DOAK kann nur Dabigatran dialysiert werden. Der Dabigatran-Plasmaspiegel kann bei Patienten mit akutem Nierenversagen durch Hämodialyse gesenkt werden, und ein erfolgreicher Einsatz dieses Verfahrens wurde bei schweren Blutungen unter Dabigatran beschrieben [65, 66]. Praktische Aspekte zur Hämodialyse bei Blutungen unter Dabigatran sind in Tab. 5 zusammengefasst.

Der mögliche Nutzen, die Machbarkeit, aber auch das Risiko (Blutungsrisiko bei Anlage eines Hämofiltrationskatheters, Verzögerung des operativen Eingriffs um Stunden) einer Hämodialyse sollten in Erwägung gezogen werden.

Das momentane Fehlen eines Antidots für DOAK mag zwar auf den ersten Blick ein Nachteil sein, jedoch spiegelt die klinische Erfahrung aus den Studien dies nicht wider. Aus der RE-LY Studie ist bekannt, dass die 30-Tages-Mortalität nach schwerer Blutung unter Dabigatran sogar tendenziell besser war als unter VKA $(p=0,052)[13,47]$. Ähnliche Daten liegen auch für Rivaroxaban vor. Das fehlende Antidot sollte daher kein Argument gegen eine Therapie mit DOAK sein, zumal die Rate an intrazerebralen Blutungen für alle Substanzen niedriger war als unter VKA.

Derzeit ist eine großangelegte internationale Studie mit einem ersten Antidot (Idarucizumab; ein humanisierter muriner Antikörper) gegen Dabigatran in konkreter Planung. Die Wirkung erfolgt binnen weniger Minuten und ist damit wesentlich schneller als jedes Mittel gegen Blutungen unter VKA. Experten gehen davon aus, dass das Vorhandensein eines Antikörpers, sofern sich dessen Effektivität und Sicherheit bestätigt, ein wesentlicher Schritt in die Akzeptanz von DOAK sein wird. 


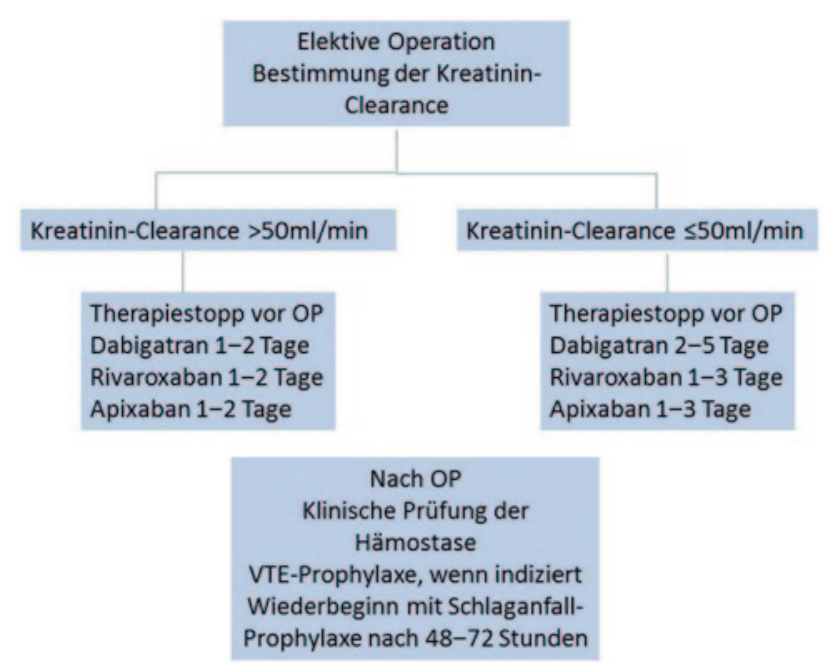

Abb. 1 Vorschläge für den Zeitpunkt des Absetzens von DOAK vor elektivem Eingriff. (Quellen: modifiziert nach [8, 34, $60,67])$

\section{Perioperatives Management}

Für das oft auch als „Bridging“ bezeichnete perioperative Management von mit VKA antikoagulierten Patienten gibt es Leitlinien, auf die in diesem Zusammenhang nicht näher eingegangen wird $[19,20]$.

Bei den DOAK ist ein präoperatives „Bridging“ aufgrund der kurzen Halbwertszeit meist nicht erforderlich, außer in bestimmten Situationen, z. B. bei Verschiebungen von operativen Eingriffen bei Hochrisikopatienten. Rivaroxaban und Apixaban können bis 1-2 Tage vor der Operation verabreicht werden (siehe dazu die jeweilige Fachinformation) [2, 3, 4]. Der Zeitpunkt des Absetzens hängt vor allem bei Dabigatran wegen der hohen renalen Ausscheidungsrate von der Nierenfunktion und dem Blutungsrisiko der Operation ab. Ein sehr einfaches, an verschiedene Publikationen angelehntes Schema als Vorschlag für das perioperative Management ist in Abb. 1 angeführt [8, 60, 67]. 2013 wurde von der „European Heart Rhythm Association“ (EHRA) ein praxisorientierter Leitfaden publiziert, der die meisten Aspekte des Umgangs mit DOAK (so auch das perioperative Management) abdeckt [34].

Teilweise unterscheiden die Empfehlungen zwischen Operationen mit hohem und niedrigem Blutungsrisiko. Der Evidenzgrad für all diese zum Teil auch unterschiedlichen Empfehlungen ist niedrig; sie beruhen in der Regel auf Expertenmeinungen. Gegebenenfalls kann mittels Gerinnungstests (z. B. mittels aPTT) das Abklingen der Wirkung von DOAK dokumentiert werden (siehe Punkt Labor und DOAK dieses Konsensus). Für das perioperative Vorgehen bei Patienten unter Dabigatran wurden bereits österreichische Konsensusempfehlungen publiziert [25], und auch in den Fachinformationen wird auf diese Situationen Bezug genommen [2, 3, 4].

Wenn eine Indikation für eine postoperative Thromboseprophylaxe in einer zugelassenen Indikation (elektiver Hüft- oder Kniegelenksersatz) mit einem DOAK gegeben ist, so sollte dies streng nach den Vorgaben (prophylaktische Dosierung) erfolgen. Alternativ kann die übliche VTE-Prophylaxe durchgeführt werden, was meist ca. $6 \mathrm{~h}$ nach der OP der Fall ist, bis das DOAK wieder in therapeutischer Dosis verabreicht werden kann. Die Wiedereinleitung einer therapeutischen Dosis von $\mathrm{NMH}$, VKA oder DOAK in Abhängigkeit vom Operationstyp, der Nachblutungsgefahr, dem Thromboserisiko und dem postoperativen Verlauf wird frühestens nach 48-72 h empfohlen.

Zusammenfassung: Bei den DOAK ist präoperatives Bridging mit Heparin in der Regel nicht erforderlich. Wenn eine Indikation für die postoperative Prophylaxe in einer zugelassenen Indikation (z. B. Hüft- oder Kniegelenksersatz) mit einem DOAK gegeben ist, so kann diese auch mit einem DOAK in der postoperativ zugelassenen Dosierung durchgeführt werden. Alternativ sollte die übliche VTE-Prophylaxe (zumeist mit NMH) erfolgen, bis das DOAK wieder in therapeutischer Dosis verabreicht werden kann.

\section{Spezifische neurologische Aspekte bei Verwendung von DOAK}

Es sind mehrere klinisch relevante Fragestellungen im Rahmen der Akutversorgung bei TIA/Schlaganfall von Bedeutung:

a. Thrombolyse des ischämischen Schlaganfalls bei bekannter bzw. nicht bekannter Therapie mit VKA/ DOAK

b. Beginn einer Therapie mit VKA/DOAK nach TIA/nach ischämischem Schlaganfall

c. Management der intrazerebralen Blutung bei Therapie mit VKA/DOAK

d. Therapie mit VKA/DOAK nach intrazerebraler Blutung

Ad a) Thrombolyse des ischämischen Schlaganfalls bei bekannter bzw. nicht bekannter Therapie mit VKA/ DOAK Wenn ein Patient mit einem VKA vorbehandelt ist, dann wird die Entscheidung zur systemischen Thrombolyse individuell getroffen: Nach Zulassung der FDA ist eine systemische Thrombolyse bei einer INR $<1,7$ möglich. Die EMA betrachtet eine Vorbehandlung mit VKA als Kontraindikation für eine systemische Thrombolyse, d. h. die INR sollte $<1,3$ sein. Das Einblutungsrisiko in den Infarkt ist erhöht, wenn die systemische Thrombolyse bei einer INR zwischen 1,3 und 1,7 durchgeführt wird. Es gibt aber keine Hinweise in der Literatur dafür, dass das erhöhte Einblutungsrisiko das funktionelle Ergebnis oder die Mortalität nach drei Monaten beeinflusst [57].

Bei Vorbehandlung mit einem DOAK müssen quantitative, Substanz-spezifische Gerinnungsanalysen durchgeführt werden. Für Dabigatran gilt laut Zulassung: „Der Gebrauch von Fibrinolytika zur Behandlung des akuten 
ischämischen Schlaganfalls kann bei Patienten, die vor Beginn der Behandlung eine quantitative Bestimmung der Thrombinzeit in verdünnten Plasmaproben, EcarinClotting-Zeit oder aPTT innerhalb des Normbereichs bezogen auf die jeweiligen laborspezifischen Normwerte aufweisen, in Erwägung gezogen werden.“

Für Faktor Xa-Hemmer gilt: Für Rivaroxaban (und angekündigt auch für Apixaban) gibt es einen geeichten Anti-Faktor-Xa-Test. In vielen Labors zeitnah verfügbar ist der für niedermolekulares Heparin geeichte Anti-Faktor-Xa-Test. Wenn keine Faktor-Xa-Hemmung in einem dieser Tests vorliegt, kann ein klinisch relevanter Wirkspiegel von Xabanen mit hoher Wahrscheinlichkeit ausgeschlossen und die Durchführung einer systemischen Thrombolyse erwogen werden.

Gibt es an der versorgenden Einrichtung keine Substanz-spezifischen Gerinnungsanalysen (Thrombinzeit, Hemoclot $^{\mathrm{rm}}$-Test, Tests auf Faktor-Xa-Hemmung), dann sollte eine systemische Thrombolyse nicht durchgeführt werden, wenn eine Therapie mit einem DOAK besteht und dessen letzte Einnahme innerhalb der vergangenen $48 \mathrm{~h}$ erfolgte (analoges Vorgehen $\mathrm{zu}$ operativen Eingriffen).

Grundsätzlich gilt, dass bei Kontraindikationen für eine systemische Thrombolyse aufgrund einer vorliegenden oralen Antikoagulation eine endovaskuläre Therapie (Thrombektomie) erwogen werden kann, wenn ein proximaler Verschluss einer der großen Hirnarterien vorliegt.

Ad b) Beginn einer Therapie mit VKA/DOAK nach TIA/ nach ischämischem Schlaganfall Zur Frage, wann nach einer TIA bzw. nach einem ischämischen Schlaganfall die orale Antikoagulation mit einem VKA begonnen werden kann, liegen allgemein akzeptierte Empfehlungen vor: Bei TIA bzw. einem „Minor Stroke“ (Schweregrad nach der NIHSS-Skala <4) kann innerhalb von 14 Tagen mit der Einstellung auf VKA begonnen werden. Diese Empfehlung der AHA basiert auf der EAFT-Studie [21]. Die europäische Schlaganfall-Organisation (ESO) hat in ihrer Empfehlung sogar die Möglichkeit des sofortigen Beginns der Einstellung auf VKA angegeben [55]. Voraussetzung ist, dass der Blutdruck gut eingestellt ist und keine Zeichen einer Einblutung in den Infarkt bestehen. Bei einem schweren ischämischen Schlaganfall (NIHSS Skala $>4$ ) wird empfohlen, die Einstellung auf VKA auf die Zeit nach zwei Wochen (AHA) bzw. nach vier Wochen (ESO) zu verschieben. Auch hier wird erst eine neue Bildgebung durchgeführt, um vor der Einstellung eine Einblutung in das Infarktareal auszuschließen.

Da im Unterschied zu den VKA bei DOAK die maximale Antikoagulation bereits nach ca. zwei Stunden erreicht wird, keine Möglichkeit der Antagonisierung besteht und auch keine Studienerfahrung mit dem frühzeitigen Einsatz der DOAK vorliegt, erscheint folgende Vorgangsweise sinnvoll: Bei einer TIA ohne Gewebsläsion (negativem DWI-MR) kann die Therapie mit einem DOAK sofort begonnen werden. Bei TIA mit Gewebsläsion (positivem DWI-MR) oder „Minor Stroke“ (NIHSS-
Score $<4$ ) kann ein DOAK nach 3-14 Tagen verabreicht werden, jedoch erst nach neuerlicher Bildgebung, um eine parenchymatöse Einblutung auszuschließen. Liegt eine solche vor, wird der Beginn der OAK bis zur Resorption der Blutung verschoben. Bei „Major Stroke“ (NIHSS-Score $>4$ ) kann nach 14 bis 28 Tagen und erneuter Bildgebung zum Ausschluss einer parenchymatösen Blutung mit DOAK begonnen werden. Risikofaktoren für eine symptomatische Einblutung sind die Größe des Infarkts, Kardioembolie, das Alter des Patienten, Begleiterkrankungen wie Hypertonie, Diabetes mellitus oder früherer Schlaganfall, zerebrale Mikroangiopathie sowie petechiale Einblutungen in das ischämische Areal.

Ad c) Management der intrazerebralen Blutung bei Therapie mit VKA/DOAK Der Begriff „intrakranielle Blutung" umfasst die verschiedensten Arten von Blutungen innerhalb der Schädelkalotte wie subdurale, subarachnoidale, intrazerebrale und (sehr selten) intraventrikuläre Blutungen. Die Behandlung der (meist traumatischen) subduralen Blutungen sowie der (meist aneurysmatischen) subarachnoidalen Blutungen ist individuell und interdisziplinär festzulegen. Intrazerebrale Blutungen können weiter unterteilt werden in primäre und sekundäre intrazerebrale Blutungen. Die Blutung ist „primär“, wenn die Ursache der Einriss in der Gefäßwand ist. Die Blutung wird als „,sekundär“ bezeichnet, wenn das primäre Ereignis ein Gefäßverschluss ist und sekundär eine Einblutung entsteht. Die Begriffe „primäre intrazerebrale Blutung“ und „hämorrhagischer Schlaganfall“ werden synonym verwendet. Von 100 Personen, die einen Schlaganfall erleiden, ist bei $85 \%$ die Ursache primär ischämisch und bei $15 \%$ primär eine Blutung (d. h. hämorrhagischer Schlaganfall oder primäre intrazerebrale Blutung). Ca. $50 \%$ der primären intrazerebralen Blutungen sind Folge einer hypertensiv bedingten zerebralen Mikroangiopathie und ca. $20 \%$ die Folge einer zerebralen Amyloidangiopathie (CAA). Plättchenhemmer haben eine zunehmend beachtete Rolle bei der Entstehung dieser Blutungen. Bei ca. 15\% der Patienten mit einer intrazerebralen Blutung ist die Antikoagulation (bisher VKA) als primäre Ursache anzusehen, wobei ca. $80 \%$ der Ereignisse bei einer INR im Zielbereich und ca. $20 \%$ der Ereignisse bei Überdosierung der VKA auftreten. Selten (5\%) sind vaskuläre Malformationen (arteriovenöse Malformationen, Aneurysmen, Kavernome) die Ursache einer primären intrazerebralen Blutung.

Blutungen als Folge einer oralen Antikoagulation mit VKA haben eine schlechte Prognose mit einer Mortalität von ca. $60 \%$. Die Mortalität ist hoch, obwohl die Wirkung der VKA an der versorgenden Schlaganfall-Einheit rasch aufgehoben werden kann. Gegenwärtiger Standard bei der Behandlung einer intrazerebralen Blutung unter VKA ist: Gabe von PCC (25-50 IE/kg KG) und - zusätzlich - intravenöse Gabe von Vitamin K (z. B. 10-20 mg langsam - als Kurzinfusion). Der Blutdruck wird in dieser Situation rasch (innerhalb von $60 \mathrm{~min}$ ) auf Werte unter 140/90 mmHg gesenkt. 
Tab. 6 Vorgehen bei intrazerebraler Blutung unter DOAK. (Quelle: Autoren)

\begin{tabular}{l}
\hline Absetzen des DOAK und anderer gerinnungshemmender Medikamente \\
\hline Blutdruck-Management (wie bei VKA), Ziel: RR $<140 / 90 \mathrm{mmHg}$ \\
\hline Diurese fördern (Flüssigkeitszufuhr) \\
\hline Analyse der Gerinnung, wenn möglich quantitative Substanzbestimmung \\
\hline Wenn ein gerinnungshemmender Effekt anzunehmen bzw. nachweisbar ist, \\
Gabe von PCC (50 IE/kg KG) oder rFVlla ( $90 \mu \mathrm{gg} / \mathrm{kg}$ KG) \\
\hline Gabe von Thrombozyten bei Thrombozytopenie; bei Vortherapie mit einem \\
Plättchenhemmer auch bei normaler Thrombozytenzahl \\
\hline Hämodialyse/Hämofiltration bei Dabigatran erwägen
\end{tabular}

Das Risiko einer intrazerebralen Blutung unter DOAK ist signifikant geringer als das Risiko einer intrazerebralen Blutung unter VKA; es lag in den großen Studien bei ca. $0,3 \%$ und war vergleichbar mit dem Risiko einer intrazerebralen Blutung bei Gabe eines Plättchenhemmers (AVERROES-Studie mit Apixaban). Anzumerken ist, dass das niedrige intrazerebrale Blutungsrisiko von ca. 0,3\% unter DOAK für ein mittleres Alter von 72 Jahren und bei gutem Risikomanagement (insbesondere bei guter Blutdruckbehandlung) angenommen werden kann. Bei hohem Lebensalter und zerebrovaskulären Vorerkrankungen ist das Risiko wohl höher. Bisher wurde die Mortalität bei intrakraniellen Blutungen (subdural, subarachnoidal und intrazerebral) unter Einnahme von DOAK nur für Dabigatran veröffentlicht. Hier zeigte sich kein signifikanter Unterschied der Mortalität im Vergleich zu den VKA. 25 der ca. 12.000 Personen, die über zwei Jahre mit Dabigatran behandelt wurden, erlitten eine intrazerebrale Blutung. 16 der 25 Personen verstarben (Mortalität bei $60 \%$ ). Bei VKA erlitten 46 Personen eine intrazerebrale Blutung, 19 der 46 Personen verstarben $(41 \%)$.

Tabelle 6 zeigt ein mögliches Vorgehen bei einer intrakraniellen (subduralen, subarachnoidalen oder intrazerebralen) Blutung unter DOAK.

Ad d) Therapie mit VKA/DOAK nach intrazerebraler Blutung Die Ursache einer intrazerebralen Blutung stellt eine wichtige Entscheidungshilfe dar und sollte geklärt werden. Bei Zustand nach einer Hypertonie-bedingten intrazerebralen Blutung liegt das Risiko für eine erneute intrazerebrale Blutung unter oraler Antikoagulation mit einem VKA bei $2 \%$ pro Jahr, wenn der Blutdruck gut eingestellt ist [22]. Da DOAK ein niedrigeres Risiko für intrazerebrale Blutungen haben, ist bei Einstellung auf ein DOAK von einem niedrigeren Rezidivrisiko auszugehen als bei Blutung unter VKA. Voraussetzung ist eine gute antihypertensive Therapie, wie sie in allen klinischen Studien erfolgte. Je nach embolischem Risiko ( $\mathrm{CHA}_{2} \mathrm{DS}_{2}$-VASc-Score) wird durch Abwägung von Nutzen und Risiko die Entscheidung getroffen.

Nach einer intrazerebralen Blutung infolge einer Amyloidangiopathie liegt das jährliche Risiko für eine Blutung unter oraler Antikoagulation mit VKA bei $11 \%$ [22], was als Kontraindikation für eine neuerliche Antikoagulation zu sehen ist.
Handelte es sich um eine intrazerebrale Blutung unter OAK ohne Risikofaktoren, dann liegt das jährliche Risiko für eine erneute intrazerebrale Blutung unter OAK bei $5 \%[46]$.

Die Wiedereinstellung eines Patienten auf OAK nach einer zerebralen Blutung bedarf äußerst kritischer Abwägung. Überlegungen hierzu sind in Tab. 9 angeführt.

Nach einer intrazerebralen Blutung unter OAK sollte ca. drei bis fünf Monate gewartet werden, bevor man wieder mit einer OAK beginnt.

\section{Scoring-Systeme zur Evaluierung der Thromboembolierisikos}

Zur Beurteilung des Thromboembolierisikos ziehen die Autoren in Übereinstimmung mit der ESC den $\mathbf{C H A}_{2} \mathbf{D S}_{2}-$ VASc-Score heran. Die ESC hat sich für die Verwendung dieses Scores (Tab. 7) ausgesprochen, da er im Vergleich mit anderen Risikoscores den höchsten Anteil an Hochrisikopatienten mit VHF identifiziert [43] und vor allem

Tab. 7 Der $\mathrm{CHA}_{2} \mathrm{DS}_{2}$-VASc-Score. (Quelle: [43])

\begin{tabular}{|c|c|}
\hline Parameter & Punkte \\
\hline Herzinsuffizienz $z^{\mathrm{a}}$ & 1 \\
\hline Hypertonie $^{\mathrm{a}}$ & 1 \\
\hline \multicolumn{2}{|l|}{ Alter: } \\
\hline$<65 a$ & 0 \\
\hline $65-74 a$ & 1 \\
\hline$\geq 75 a$ & 2 \\
\hline Diabetes mellitus & 1 \\
\hline Insult/TIA/Thromboembolie ${ }^{a}$ & 2 \\
\hline $\begin{array}{l}\text { Arterielle Gefäßerkrankung (Myokardinfarkt, pAVK, } \\
\text { Aortenplaques) }\end{array}$ & 1 \\
\hline Weibliches Geschlecht & 1 \\
\hline \multicolumn{2}{|c|}{$\begin{array}{l}\text { aaktuell oder anamnestisch } \\
\text { Bei } 0 \text { Punkten (Männer) oder } 1 \text { Punkt (Frauen ohne weiteren Risikofaktor) } \\
\text { liegt ein sehr niedriges, bei } 1 \text { Punkt (Männer) ein niedriges bis mittleres } \\
\text { Risiko und ab } 2 \text { Punkten (Männer und Frauen) ein mittleres bis hohes Risiko } \\
\text { für das Auftreten eines Insults bei VHF vor }\end{array}$} \\
\hline
\end{tabular}

Tab. 8 Insultrisiko nach $\mathrm{CHA}_{2} \mathrm{DS}_{2}$-VASc-Punktezahl. (Quelle: [12])

\begin{tabular}{|l|l|l|}
\hline $\mathrm{CHA}_{2} \mathrm{DS}_{2}$-VASc-Punkte & Patientenzahl $(n=73.538)$ & $\begin{array}{l}\text { Korrigierte Insultrate } \\
(\% \text { pro Jahr })\end{array}$ \\
\hline 0 & 6.369 & 0,78 \\
\hline 1 & 8.203 & 2,01 \\
\hline 2 & 12.771 & 3,71 \\
\hline 3 & 17.371 & 5,92 \\
\hline 4 & 13.887 & 9,27 \\
\hline 5 & 8.942 & 15,26 \\
\hline 6 & 4.244 & 19,74 \\
\hline 7 & 1.420 & 21,50 \\
\hline 8 & 285 & 22,38 \\
\hline 9 & 46 & 23,64 \\
\hline
\end{tabular}


bei Berücksichtigung der Antikoagulationsempfehlungen, anders als der CHADs-Score, kosteneffektiv ist.

Tabelle 8 zeigt die Zuordnung der $\mathrm{CHA}_{2} \mathrm{DS}_{2}$-VAScPunktezahl zum Insultrisiko anhand der aktuellsten Daten der ESC [12].

$\mathrm{Zu}$ bemerken ist, dass in allen DOAK-Schlüsselstudien (RE-LY, ROCKET-AF, ARISTOTLE, ENGAGE) der Patienteneinschluss nicht nach dem $\mathrm{CHA}_{2} \mathrm{DS}_{2}$-VASc-Score erfolgte, sondern nach jeweils etwas anders definierten Risikokonstellationen. Der in diesen Studien noch verwendete ältere CHADs-Score wurde in der Regel jeweils erst retrospektiv berechnet.

Die österreichischen Experten sprechen sich, in Übereinstimmung mit der ESC, für die Verwendung des $\mathrm{CHA}_{2} \mathrm{DS}_{2}$-VASc-Scores aus.

\section{Österreichische Konsensusempfehlungen zur Thromboembolie-Prophylaxe bei nicht-valvulärem Vorhofflimmern}

Die derzeit am weitesten beachteten Leitlinien sind die des „American College of Chest Physicians“ (ACCP) und die der „European Society of Cardiology“ (ESC). Die Österreichische Kardiologische Gesellschaft hat sich grundsätzlich dazu entschlossen, die ESC-Leitlinien anzuwenden.

Das 2012 publizierte Update der ESC-Leitlinien zum nicht-valvulären VHF [11] enthält eine ganze Reihe neuer bzw. modifizierter Empfehlungen. (Valvuläres Vorhofflimmern ist definiert als VHF bei Patienten mit einer postrheumatischen Mitralklappenstenose oder bei Patienten mit mechanischem Herzklappenersatz [10, 11]. Bei valvulärem VHF ist ausschließlich eine Antikoagulation mit VKA indiziert $[10,11]$.)

Unter anderem wurde erstmals auch das asymptomatische VHF erfasst. Es konnte gezeigt werden, dass das Vorliegen von atrialen Tachyarrhythmien (z. B. Vorhofflattern) bei Patienten ohne klinisch apparentes VHF das Risiko für ischämischen Insult oder systemische Embolie signifikant erhöht [33]. Es wird deshalb bei Patienten ab einem Alter von 65 Jahren ein VHF-Screening mittels Pulsmessung plus EKG empfohlen, um VHF möglichst früh zu diagnostizieren (1B) [11].

Die rezenten ESC Leitlinien berücksichtigen bereits umfassend die DOAK und stellen die Basis für die Empfehlungen der österreichischen Konsensusgruppe dar, die im folgenden Kapitel dargelegt sind.

Auf Basis des $\mathrm{CHA}_{2} \mathrm{DS}_{2}$-VASc-Scores und basierend auf den ESC-Leitlinien werden folgende Empfehlungen gegeben. Spezielle Überlegungen der österreichischen Konsensusgruppe werden in „Kommentaren“ dargelegt.

CHA DS $_{2}$-VASc-Score 0: VHF-Patienten mit niedrigem Risiko $\left(\mathrm{CHA}_{2} \mathrm{DS}_{2}\right.$-VASc-Score 0$)$ - das sind per definitionem Patienten unter 65 Jahren ohne weitere Risikofaktoren - sind mit keiner antithrombotischen Therapie zu behandeln. Dies gilt auch für Frauen, die lediglich aufgrund ihres Geschlechts einen $\mathrm{CHA}_{2} \mathrm{DS}_{2}$ VASc-Score von 1 aufweisen.

$\mathbf{C H A}_{2} \mathbf{D S}_{2}$-VASc-Score 1: Bei männlichen Patienten mit einem $\mathrm{CHA}_{2} \mathrm{DS}_{2}$-VASc-Score von 1 sollte eine OAK mit einem VKA (INR 2-3), einem direkten Thrombininhibitor (Dabigatran) oder einem oralen FaktorXa-Inhibitor (Rivaroxaban, Apixaban) auf Basis der Einschätzung des Blutungsrisikos und der Patientenpräferenzen erwogen werden (IIaA - „sollte erwogen werden") [11].

$\mathbf{C H A}_{2} \mathbf{D S}_{2}$-VASc-Score $\geq 2$ : Bei Patienten beiden Geschlechts mit einem $\mathrm{CHA}_{2} \mathrm{DS}_{2}$-VASc-Score von $\geq 2$ sollte eine OAK mit einem VKA (INR 2-3), einem direkten Thrombininhibitor (Dabigatran) oder einem oralen Faktor-Xa-Inhibitor (Rivaroxaban, Apixaban) verabreicht werden (1A - „wird empfohlen“) [11].

Bei Patienten, die eine OAK - sei es mit VKA oder DOAK - trotz ausführlicher Aufklärung verweigern, sollte eine Therapie mit Thrombozytenfunktionshemmern erwogen werden, und zwar entweder, bei niedrigem Blutungsrisiko, eine Dualtherapie mit 75-100 mg ASS plus 75 mg Clopidogrel pro Tag oder, bei erhöhtem Blutungsrisiko und mit geringerer Wirksamkeit, eine Monotherapie mit 75-325 mg ASS pro Tag (IIaB- Empfehlung) [11].

Neueinstellung: Wenn bei einem VHF-Patienten eine OAK indiziert ist, sollte in den meisten Fällen aufgrund des klinischen Nettonutzens ein direkter Thrombininhibitor (Dabigatran) oder ein oraler Faktor-Xa-Inhibitor (Rivaroxaban, Apixaban) einem VKA vorgezogen werden (IIaA - Empfehlung) [11].

Kommentar: Für die Praxis ist wichtig, dass zunächst entschieden werden muss, ob bei einem Patienten eine Indikation für eine OAK besteht oder nicht. Erst in einem zweiten Schritt kann dann entschieden werden, ob ein VKA oder ein DOAK verabreicht wird.

Da in den ESC-Leitlinien für Männer bei einem $\mathrm{CHA}_{2} \mathrm{DS}_{2}$-VASc-Score von 1 eine OAK erwogen werden sollte (IIaA) und bei Indikation für eine OAK einem DOAK der Vorzug gegenüber einem VKA gegeben werden sollte (IIaA), würde dies bedeuten, dass einer großen Zahl von Patienten mit niedrigem Risiko, die bisher keine antithrombotische Therapie erhalten haben oder auf Plättchenfunktionshemmer eingestellt waren, die neue Substanzen verschrieben werden sollten. Derzeit stehen dieser Empfehlung, die wahrscheinlich zu einer Abnahme von Schlaganfällen und den entsprechende Folgekosten führen würde, Verschreibungsvorgaben (z. B. dass DOAK nur angewandt werden können, wenn VKAs nicht im therapeutischen Bereich gehalten werden können oder mit Nebenwirkungen verbunden sind) in Österreich, aber auch in anderen Ländern, gegenüber. 
Ein Vergleich des DOAK Apixaban mit ASS in der AVERROES-Studie bei Patienten, bei denen kein VKA gegeben werden konnte, zeigte eine signifikant bessere Wirksamkeit von Apixaban gegenüber ASS bei vergleichbaren Blutungsraten [53]. Plättchenfunktionshemmer stellen daher keine adäquate Therapie bei Patienten mit Vorhofflimmern dar und sollten als obsolet betrachte werden (Ausnahme siehe $\mathrm{CHA}_{2} \mathrm{DS}_{2}$-VASc-Score $>2$ )

Umstellung: Wenn VKA bei einem VHF-Patienten, bei dem eine OAK indiziert ist, wegen schlechter Einstellbarkeit, Nebenwirkungen oder Problemen, das INR-Monitoring einzuhalten, nicht verwendet werden können, wird die Verwendung eines direkten Thrombininhibitors (Dabigatran) oder eines oralen FaktorXa-Inhibitors (Rivaroxaban, Apixaban) empfohlen (IB - Empfehlung [11].

Bei gut auf VKA eingestellten und stabilen Patienten wird eine Umstellung auf ein DOAK nicht als vorrangig gesehen, obwohl die Empfehlung auch hier lautet, DOAK zu bevorzugen.

Kommentar: Was gut auf VKA eingestellte Patienten angeht, so stehen viele Fachgesellschaften und Experten (z. B. [53]) derzeit auf dem Standpunkt, dass diese Patienten nicht aktiv auf DOAK umgestellt werden müssen - ein Standpunkt, dem sich auch die überwiegende Zahl der Autoren dieses Konsensus anschließt.

Zur Frage, ob Patienten, die gut auf einen VKA eingestellt sind, aber ein hohes Blutungsrisiko aufweisen, jedoch bisher nie geblutet haben, auf ein DOAK umgestellt werden sollen oder nicht, sind die Meinungen geteilt.

Bezüglich der Frage, wann ein Patient von einem VKA auf ein DOAK umgestellt werden soll, ist die Qualität der Einstellung auf einen VKA von Bedeutung. Die Frage, wann ein Patient "gut eingestellt" ist, ist keineswegs einfach zu beantworten, aber es wird allgemein akzeptiert, dass von zehn INR-Messungen mindestens sieben im therapeutischen Bereich sein müssen um eine "gute“ Einstellung zu dokumentieren.

$\mathrm{Zu}$ bedenken ist allerdings, dass auch bei konstant im therapeutischen Bereich unter VKA eingestellten Patienten das intrakranielle Blutungsrisiko im Vergleich $\mathrm{zu}$ DOAK höher ist [64]. Dies dürfte daran liegen, dass VKA eine Wirkung auf den im Gehirn in hoher Konzentration vorliegenden „tissue factor“, der die lokale Homöostase kontrolliert, ausüben [37, 61].

\section{Antithrombotische Kombinationstherapie}

Grundsätzlich ist bei jeder Antikoagulation das Thromboembolierisiko gegen das Blutungsrisiko abzuwägen. Dies gilt besonders auch für die antithrombotische Kombinationstherapie. In einer Kohortenstudie mit insgesamt 82.854 VHF-Patienten, die nach einer Hospitalisierung wegen VHF eine antithrombotische Therapie erhielten, lag die Gesamt-Blutungsrate (einschließlich tödlicher
Blutungsereignisse) in einer Beobachtungszeit von 3,3 Jahren bei 11,4\% [32]. „Dual“- oder "Triple“-Therapien zeigten höhere Blutungsraten als Monotherapien mit VKA, ASS oder Clopidogrel, wobei die Dualtherapie VKA/ Clopidogrel eine Hazard Ratio (HR) von 3,08, die Tripletherapie VKA/ASS/Clopidogrel eine HR von 3,70 ergab.

Eine andere Studie untersuchte die Mortalitätsraten bei Patienten mit koronaren Stents, die sowohl eine duale Antiplättchentherapie als auch einen VKA benötigten [56]. Dabei zeigte sich, dass - im Vergleich zu Patienten, die lediglich eine duale Antiplättchentherapie erhielten - die Mortalität unter „Triple“-Therapie dann nicht erhöht war, wenn die INR unter 2,6 lag. Hingegen war bei „Triple“-Therapie und einer INR $\geq 2,6$ die Mortalität über 18 Monate signifikant höher (blutungsfreies Überleben 95,1 vs. 66,7\%).

Ein Konsensus der ESC, gemeinsam mit zwei anderen Fachgesellschaften, der „European Heart Rhythm Association“ (EHRA) und der „European Association of Percutaneous Cardiovascular Interventions" (EAPCI) gibt Empfehlungen für die Dauer der „Triple“-Therapie bei Patienten mit VHF und KHK [44, 45]. Anzumerken ist, dass diese bereits 2010 veröffentlichten europäischen Empfehlungen DOAK als Alternative zu VKA noch nicht berücksichtigen, während die nordamerikanischen Empfehlungen von 2011 bereits Dabigatran $(2 \times 110 \mathrm{mg} / \mathrm{die})$ als möglichen Ersatz für VKA nannten [24]. Weiters gibt es erste Daten dafür, dass die duale Antiplättchentherapie bei modernen DES der zweiten und dritten Generation wahrscheinlich verkürzt werden kann [62]. Die optimale Dauer der dualen Antiplättchentherapie nach Stentimplantation ist derzeit noch nicht gesichert. In einem Positionspapier („Practical Guide“) der „European Heart Rhythm Association" wird es als vertretbar erachtet, dass Patienten, bei denen schon vor der koronaren Intervention DOAK gegenüber VKA als vorteilhafter gesehen wurden, auch nach der Intervention dasselbe Antikoagulans erhalten. Da zumindest eine plättchenhemmende Substanz zusätzlich gegeben werden muss, könnte eine niedrigere Dosis des Antikoagulans eine sicherere Option darstellen [35]. Nach einem AKS kann der Einsatz von DOAK zusätzlich zu einer dualen Antiplättchentherapie noch nicht empfohlen werden [16]. In einer Metaanalyse von sieben RCT wurde gezeigt, dass die Kombination eines DOAK mit Antiplättchentherapie (mono oder dual) bei Patienten mit rezentem AKS zu einer mehr als zweifach erhöhten Rate an schweren Blutungen bei nur moderater Senkung von ischämischen Endpunkten führt [51].

\section{Antikoagulation bei Kardioversion und Ablation}

\section{Kardioversion}

VKA

Bei Patienten, bei denen die VHF-Episode länger als $48 \mathrm{~h}$ andauert, ist eine OAK im therapeutischen Bereich mit einem VKA durch zumindest drei Wochen vor der Kardioversion und durch zumindest vier Wochen nach der Kardioversion indiziert (therapeutischer Bereich: INR 


\section{konsensus bericht}

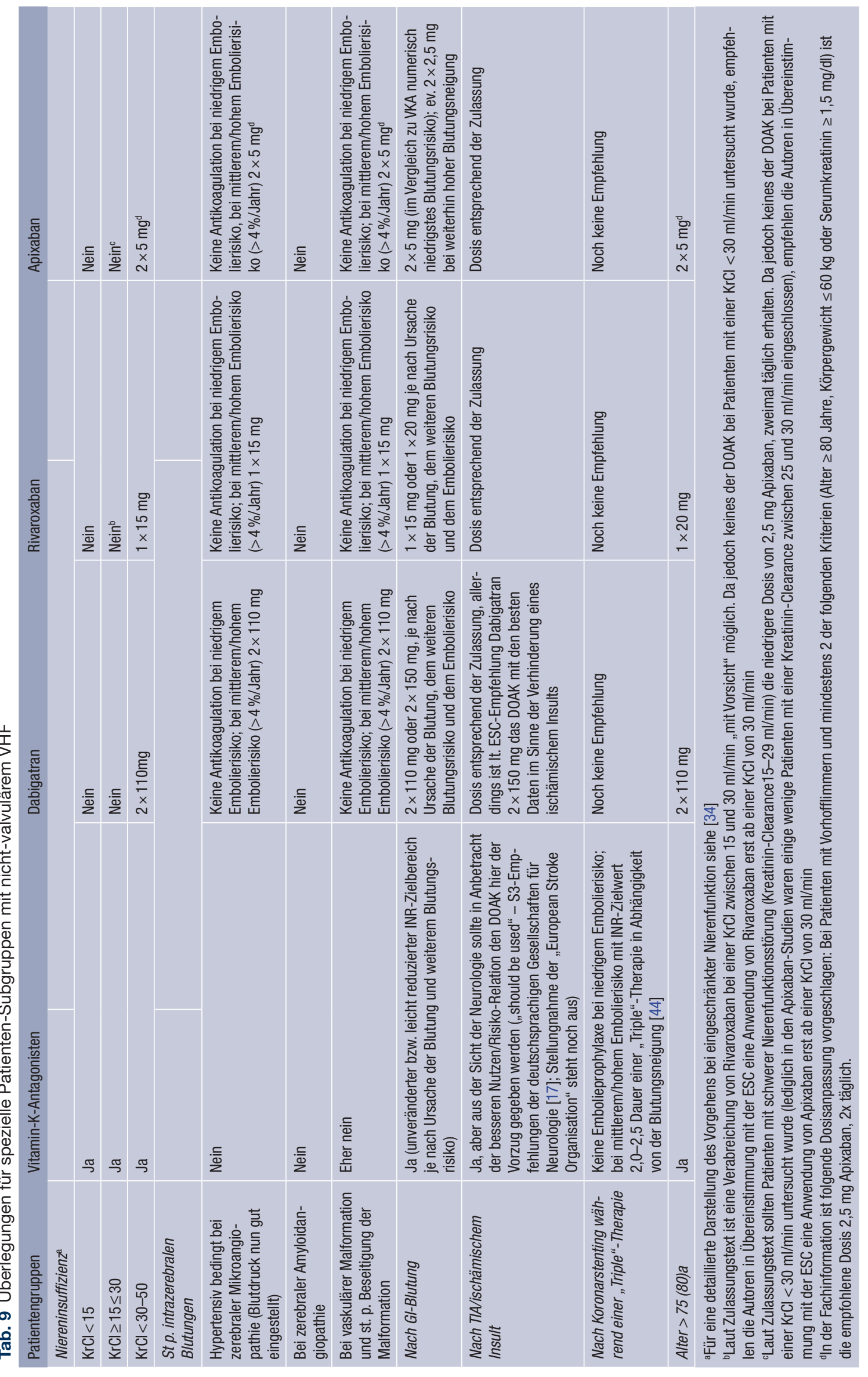


2,0-3,0, ideal 2,5-3,0), sollte sich aufgrund der individuellen Situation keine Empfehlung zu einer Langzeit-Antikoagulation ergeben $[10,11]$.

\section{DOAK}

In der RE-LY Studie, wo mehr als 18.000 Patienten mit VHF entweder mit VKA oder Dabigatran antikoaguliert wurden, wurden insgesamt fast 2.000 Patienten im Verlauf der Studie kardiovertiert [49]. Bei weniger als einem Viertel der Patienten wurde vor Kardioversion eine transösophageale Echokardiographie durchgeführt. Ein linksatrialer Thrombus konnte in 1,1-1,8\% festgestellt werden (nicht signifikant unterschiedlich für die Therapie mit Dabigatran $2 \times 110 \mathrm{mg}$, Dabigatran $2 \times 150 \mathrm{mg}$ oder VKA) Auch die Rate von Schlaganfällen oder systemischen Embolien war nach 30 Tagen insgesamt niedrig und nicht unterschiedlich in den Behandlungsgruppen. In dieser größten publizierten rezenten Kardioversionsstudie konnte somit Dabigatran als sichere und vernünftige Alternative zu VKA für die Kardioversion von VHF positioniert werden. Dementsprechend ist Dabigatran als derzeit einziges DOAK in den ESC-Guidelines von 2012 zur Kardioversion empfohlen [11].

Auch bei den knapp 300 kardiovertierten Patienten unter Rivaroxaban oder VKA in der ROCKET-AF-Studie [52] und bei den knapp 600 kardiovertierten Patienten unter Apixaban oder VKA in der ARISTOTLE-Studie [30] war das Embolierisiko nicht signifikant unterschiedlich.

\section{Pulmonalvenenisolation}

\section{VKA}

Der embolische Insult ist mit einer historischen Rate bis $3 \%$ eine der wesentlichen Komplikationen der Katheterablation zur kurativen Therapie des Vorhofflimmerns [18]. Unter einer therapeutischen (INR 2,0-2,5), für die Ablation nicht unterbrochenen Therapie mit VKA konnte diese Rate relevant gesenkt werden. Daher wird diese Strategie, mit periinterventioneller Gabe von unfraktioniertem Heparin (Activated Clotting Time - ACT - über 300), von den meisten Zentren umgesetzt [40].

\section{DOAK}

Die Problematik der Therapie mit DOAK im Zuge der Pulmonalvenenisolation ist die fehlende Erfahrung im Umgang mit Blutungskomplikationen (Perikardtamponade). In der ersten publizierten Studie wurden Patienten unter Fortsetzung der VKA-Therapie mit Patienten unter Dabigatran verglichen [42]. Die 145 DabigatranPatienten setzten nur die Morgendosis am Tag der Ablation aus und zeigten dementsprechend signifikant mehr Blutungskomplikationen (6\% im Vergleich zu $1 \%$ unter VKA). Eine weitere Studie verglich VKA und Dabigatran $2 \times 110 \mathrm{mg}$ (pausiert am Morgen der Ablation) und fand kein erhöhtes Thromboembolie- oder Blutungsrisiko [39]. Auch eine rezente Studie an mehr als 700 Patienten, bei denen Dabigatran $24 \mathrm{~h}$ vor dem Eingriff pausiert wurde, zeigte kein erhöhtes Insultrisiko und kein erhöhtes Blutungsrisiko unter Dabigatran [41]. Dem- entsprechend kann Dabigatran wohl auch im Zuge der Pulmonalvenenisolation sicher und effektiv eingesetzt werden.

Auch für die Faktor-Xa-Antagonisten, insbesondere Rivaroxaban, gibt es vorläufige Daten zur Sicherheit und Effizienz [54].

\section{Überlegungen für bestimmte Patientengruppen}

Im Folgenden werden noch Überlegungen für spezielle Patientengruppen gegeben. Diese (siehe Tab. 9) sind lediglich als Hilfestellung zu verstehen, die auf einer eingeschränkten Datenlage beruhen. Das aktuelle Risiko für Schlaganfall, Embolie und Blutung des individuellen Patienten muss in jedem Fall beachtet und als Entscheidungsgrundlage herangezogen werden.

Speziell zur Einstellung mit DOAK nach einer zerebralen Blutung vertritt das Panel die Meinung, dass wegen des niedrigeren zerebralen Blutungsrisikos unter DOAK nach einer intrazerebralen Blutung unter VKA nur DOAK eingesetzt werden sollten. Eine zerebrale Amyloidangiopathie sollte vor einer neuerlichen Antikoagulation ausgeschlossen werden.

\section{Interessenskonflikte}

IP: Honorare für einzelne Vorträge und Advisory Board Meetings der Firmen Bayer, Boehringer Ingelheim, GSK, Pfizer, Daiichi Sankyo und Sanofi-Aventis.

SE-H: Vortragshonorare von den Firmen Bayer, Boehringer Ingelheim, Pfizer und BMS; Konsulententätigkeit für die Firmen Bayer, Boehringer Ingelheim, Daiichi Sankyo und Pfizer.

\section{RG: keine.}

W-MH: Experte, Vortragender oder Mitglied wissenschaftlicher Advisory Boards für die Firmen Bayer, Boehringer Ingelheim, Bristol-Myers Squibb, Roche und Stago.

H-PH: Vortrags-Honorare von Boehringer Ingelheim, Bayer und Pfizer; Advisory BoardMeetings für Boehringer Ingelheim, Bayer, Daiichi Sankyo und Pfizer; SAVeranstaltungen für Boehringer Ingelheim, Bayer und Daiichi Sankyo.

KH: Vortrags-Honorare von Boehringer-Ingelheim, Bayer, Daiichi Sankyo und Bristol-Myers Squibb/Pfizer.

BJ: Vortragstätigkeit für Bayer.

HCK: keine.

SK-L: Honorare für einzelne Vorträge und Advisory Board Meetings der Firmen Boehringer Ingelheim, Pfizer und BMS.

PK: Honorare für Vorträge und Teilnahme an Advisory Board Meetings von Boehringer Ingelheim, Bayer, Pfizer/ BMS und Daiichi Sankyo.

WL: Advisory Board Meetings und Vortragshonorare vonBoehringer Ingelheim, Pfizer, Bayer und Daiichi Sankyo.

FXR: Vortragshonorare von und Konsulententätigkeit für Bayer, Boehringer Ingelheim, Pfizer und Daiichi Sankyo (Vortrag). 
FW: Honorare für Vorträge und/oder Beraterfunktionen von AstraZeneca, Bayer, Boehringer Ingelheim, BMS/Pfizer, Daiichi Sankyo und Lilly.

HW: Honorare für Vorträge und/oder Beraterfunktionen von AstraZeneca, Bayer, Boehringer Ingelheim, Daiichi Sankyo, Pfizer, Roche Diagnostics, Sanofi-Aventis und Takeda.

AW: Expertentätigkeit, Vorträge, Teilnahme an Advisory Board Meetings und Studien/Forschungsprojekten für Bayer, Boehringer Ingelheim, Bristol-Myers Squibb, Daiichi Sankyo, Sanofi-Aventis und Pfizer.

JW: Teilnahme an Advisory Boards fürBoehringer Ingelheim, Bayer, Pfizer, AstraZeneca, Pharma-Allergan; Honorare für Vortragstätigkeit vonBoehringer Ingelheim, Bayer, Pfizer, MSD, und Takeda.

Die technische Durchführung des Konsensusmeetings und des nachfolgenden Abstimmungsprozesses sowie die Publikation dieses Konsensusstatements wurden durch „Unresctricted Grants“ der Firmen Bayer, Boehringer Ingelheim und Pfizer an die Österreichische Gesellschaft für Hämatologie \& Onkologie (OeGHO) ermöglicht. Alle Autoren dieser Publikation haben unentgeltlich mitgearbeitet.

\section{Danksagung}

Die Autoren danken der Firma Medical Dialogue (GF: Karl Buresch) sowie Dr. med. Norbert Hasenöhrl für ihre Unterstützung bei der Koordination und Organisation dieses Konsensus-Projekts.

\section{Open Access}

Dieser Artikel unterliegt den Bedingungen der Creative Commons Attribution License. Dadurch sind die Nutzung, Verteilung, und Reproduktion erlaubt, sofern der/ die Originalautor/en und die Quelle angegeben sind.

\section{Literatur}

1. Arbeitsgruppe „Neue Orale Antikoagulanzien“ Der ÖGLKMC und der ÖQUASTA: Stellungnahme (Update Jänner 2013). http://www.oeglmkc.at/oak/index2.html.

2. Austria Codex Online. Fachinformation Eliquis Filmtabletten. www.pharmazie.com (2014).

3. Austria Codex Online. Fachinformation Pradaxa Hartkapseln. www.pharmazie.com (2014).

4. Austria Codex Online. Fachinformation Xarelto Filmtabletten. www.pharmazie.com (2014).

5. Baglin T. The role of the laboratory in treatment with new oral anticoagulants. J Thromb Haemost. 2013;1(11 Suppl):122-8.

6. Baglin T, Hillarp A, Tripodi A, et al. Measuring Oral Direct Inhibitors (ODIs) of thrombin and factor Xa: A recommendation from the subcommittee on Control of Anticoagulation of the Scientific and Standardisation Committee of the International Society on thrombosis and haemostasis. J Thromb Haemost. 2013;11:756-60.

7. Bakhru S, Laulicht B, Jiang X, et al. PER977: a synthetic small molecule which reverses over-dosage and bleeding by the new oral anticoagulants. Circulation. 2013;128:A18809.
8. Baron TH, Kamath PS, Mcbane RD. Management of antithrombotic therapy in patients undergoing invasive procedures. N Engl J Med. 2013;368:2113-24.

9. Camm AJ, Bounameaux H. Edoxaban: a new oral direct factor xa inhibitor. Drugs. 2011;71:1503-26.

10. Camm AJ, Kirchhof P, Lip GY, et al. Guidelines for the management of atrial fibrillation: the task force for the management of atrial fibrillation of the European Society of Cardiology (ESC). Eur Heart J. 2010;31:2369-429.

11. Camm AJ, Lip GY, De Caterina R, et al. 2012 focused update of the ESC Guidelines for the management of atrial fibrillation: an update of the 2010 ESC Guidelines for the management of atrial fibrillation. Eur Heart J. 2012;33:2719-47.

12. Camm AJ, Lip GY, De Caterina R, et al. Management of atrial fibrillation - ESC-Pocket Guidelines. http://www. escardio.org/guidelines-surveys/products/pocket/Pages/ AFIB.aspxc (2012).

13. Connolly SJ, Ezekowitz MD, Yusuf S, et al. Dabigatran versus warfarin in patients with atrial fibrillation. $\mathrm{N}$ Engl J Med. 2009;361:1139-51.

14. Connolly SJ, Ezekowitz MD, Yusuf S, et al. Newly identified events in the RE-LY trial. N Engl J Med. 2010;363:1875-6.

15. Connolly SJ, Wallentin L, Ezekowitz MD, et al. The longterm multicenter observational study of dabigatran treatment in patients with atrial fibrillation (RELY-ABLE) study. Circulation 2013;128:237-43.

16. De Caterina R, Husted S, Wallentin L, et al. New oral anticoagulants in atrial fibrillation and acute coronary syndromes: ESC working group on thrombosis-task force on anticoagulants in heart disease position paper. J Am Coll Cardiol. 2012;59:1413-25.

17. Deutsche Gesellschaft Für Neurologie (Dgn). S3-Leitlinie: Sekundärprophylaxe des ischämischen Insults. http://www.dgn.org/leitlinien-online-2012/inhaltenach-kapitel/2332-ll-23-2012-sekundaerprophylaxe-desischaemischen-insults.html (2012).

18. Di Biase L, Burkhardt JD, Mohanty P, et al. Periprocedural stroke and management of major bleeding complications in patients undergoing catheter ablation of atrial fibrillation: the impact of periprocedural therapeutic international normalized ratio. Circulation. 2010;121:2550-6.

19. Douketis JD. Perioperative management of patients who are receiving warfarin therapy: an evidence-based and practical approach. Blood. 2011;117:5044-9.

20. Douketis JD, Spyropoulos AC, Spencer FA, et al. Perioperative management of antithrombotic therapy: antithrombotic therapy and prevention of thrombosis, 9th ed: American college of chest physicians evidence-based clinical practice guidelines. Chest. 2012;141:e326S-50S.

21. Eaft Study Group. Secondary prevention in non-rheumatic atrial fibrillation after transient ischaemic attack or minor stroke. EAFT (European Atrial Fibrillation Trial) Study Group. Lancet. 1993;342:1255-62.

22. Eckman MH, Rosand J, Knudsen KA, et al. Can patients be anticoagulated after intracerebral hemorrhage? A decision analysis. Stroke. 2003;34:1710-6.

23. Eerenberg ES, Kamphuisen PW, Sijpkens MK, et al. Reversal of rivaroxaban and dabigatran by prothrombin complex concentrate: a randomized, placebo-controlled, crossover study in healthy subjects. Circulation. 2011;124:1573-9.

24. Faxon DP, Eikelboom JW, Berger PB, et al. Antithrombotic therapy in patients with atrial fibrillation undergoing coronary stenting: a North American perspective: executive summary. Circ Cardiovasc Interv. 2011;4:522-34.

25. Fries D, Giurea A, Gütl M, et al. Management of dabigatran-induced bleeding: expert statement. Wien Klin Wochenschr. 2013;125:721-9. 
26. Garcia D, Barrett YC, Ramacciotti E, et al. Laboratory assessment of the anticoagulant effects of the next generation of oral anticoagulants. J Thromb Haemost. 2013;11:245-52.

27. Giugliano RP, Ruff CT, Braunwald E, et al. Edoxaban versus warfarin in patients with atrial fibrillation. N Engl J Med. 2013;369:2093-104.

28. Gladstone DJ, Bui E, Fang J, et al. Potentially preventable strokes in high-risk patients with atrial fibrillation who are not adequately anticoagulated. Stroke. 2009;40:235-40.

29. Glund S, Stangier J, Schmohl M, et al. A specific antidote for dabigatran: immediate, complete and sustained reversal of dabigatran induced anticoagulation in healthy male volunteers. Circulation. 2013;128:A17765.

30. Granger CB, Alexander JH, Mcmurray JJV, et al. Apixaban versus warfarin in patients with atrial fibrillation. $\mathrm{N}$ Engl J Med. 2011;365:981-92.

31. Halbmayer WM, Weigel G, Quehenberger P, et al. Interference of the new oral anticoagulant dabigatran with frequently used coagulation tests. Clin Chem Lab Med. 2012;50:1601-5.

32. Hansen ML, Sorensen R, Clausen MT, et al. Risk of bleeding with single, dual, or triple therapy with warfarin, aspirin, and clopidogrel in patients with atrial fibrillation. Arch Intern Med. 2010;170:1433-41.

33. Healey JS, Connolly SJ, Gold MR, et al. Subclinical atrial fibrillation and the risk of stroke. N Engl J Med. 2012;366:120-9.

34. Heidbuchel H, Verhamme P, Alings M, et al. EHRA practical guide on the use of new oral anticoagulants in patients with non-valvular atrial fibrillation: executive summary. Eur Heart J. 2013;34:2094-106.

35. Heidbuchel H, Verhamme P, Alings $M$, et al. European heart rhythm association practical guide on the use of new oral anticoagulants in patients with non-valvular atrial fibrillation. Europace. 2013;15:625-51.

36. Homepage Öquasta Version 6.0. http://oequasta.at/download/publikationen/neueAkUpdate_jaenner2013.pdf (Stand Jänner 2013).

37. Horstmann S, Rizos $\mathrm{T}$, Lauseker $\mathrm{M}$, et al. Intracerebral hemorrhage during anticoagulation with vitamin $\mathrm{K}$ antagonists: a consecutive observational study. J Neurol. 2013;260:2046-51.

38. Huber K, Connolly SJ, Kher A, et al. Practical use of dabigatran etexilate for stroke prevention in atrial fibrillation. Int J Clin Pract. 2013;67:516-26.

39. Kaseno K, Naito S, Nakamura K, et al. Efficacy and safety of periprocedural dabigatran in patients undergoing catheter ablation of atrial fibrillation. Circ J. 2012;76:2337-42.

40. Kim JS, Jongnarangsin K, Latchamsetty R, et al. The optimal range of international normalized ratio for radiofrequency catheter ablation of atrial fibrillation during therapeutic anticoagulation with warfarin. Circ Arrhythm Electrophysiol. 2013;6:302-9.

41. Kim JS, She F, Jongnarangsin $\mathrm{K}$, et al. Dabigatran vs warfarin for radiofrequency catheter ablation of atrial fibrillation. Heart Rhythm. 2013;10:483-9.

42. Lakkireddy D, Reddy YM, Di Biase L, et al. Feasibility and safety of dabigatran versus warfarin for periprocedural anticoagulation in patients undergoing radiofrequency ablation for atrial fibrillation: results from a multicenter prospective registry. J Am Coll Cardiol. 2012;59:1168-74.

43. Lip GY, Frison L, Halperin JL, et al. Identifying patients at high risk for stroke despite anticoagulation: a comparison of contemporary stroke risk stratification schemes in an anticoagulated atrial fibrillation cohort. Stroke. 2010;41:2731-8.
44. Lip GY, Huber K, Andreotti F, et al. Antithrombotic management of atrial fibrillation patients presenting with acute coronary syndrome and/or undergoing coronary stenting: executive summary-a consensus document of the European Society of Cardiology Working Group on Thrombosis, endorsed by the European Heart Rhythm Association (EHRA) and the European Association of Percutaneous Cardiovascular Interventions (EAPCI). Eur Heart J. 2010;31:1311-8.

45. Lip GY, Huber K, Andreotti F, et al. Management of antithrombotic therapy in atrial fibrillation patients presenting with acute coronary syndrome and/or undergoing percutaneous coronary intervention/ stenting. Thromb Haemost. 2010;103:13-28.

46. Majeed A, Kim YK, Roberts RS, et al. Optimal timing of resumption of warfarin after intracranial hemorrhage. Stroke. 2010;41:2860-6.

47. Majeed A, Hwang HG, Connolly SJ, et al. Management and outcomes of major bleeding during treatment with dabigatran or warfarin. Circulation. 2013;128:2325-32.

48. Mani H, Hesse C, Stratmann G, et al. Ex vivo effects of low-dose rivaroxaban on specific coagulation assays and coagulation factor activities in patients under real life conditions. Thromb Haemost. 2013;109:127-36.

49. Nagarakanti R, Ezekowitz MD, Oldgren J, et al. Dabigatran versus warfarin in patients with atrial fibrillation: an analysis of patients undergoing cardioversion. Circulation. 2011;123:131-6.

50. Ogata K, Mendell-Harary J, Tachibana M, et al. Clinical safety, tolerability, pharmacokinetics, and pharmacodynamics of the novel factor Xa inhibitor edoxaban in healthy volunteers. J Clin Pharmacol. 2010;50:743-53.

51. Oldgren J, Wallentin L, Alexander JH, et al. New oral anticoagulants in addition to single or dual antiplatelet therapy after an acute coronary syndrome: a systematic review and meta-analysis. Eur Heart J. 2013;34:1670-80.

52. Patel MR, Mahaffey KW, Garg J, et al. Rivaroxaban versus warfarin in nonvalvular atrial fibrillation. N Engl J Med. 2011;365:883-91.

53. Pengo V, Crippa L, Falanga A, et al. Questions and answers on the use of dabigatran and perspectives on the use of other new oral anticoagulants in patients with atrial fibrillation. A consensus document of the Italian Federation of Thrombosis Centers (FCSA). Thromb Haemost. 2011;106:868-76.

54. Piccini JP, Stevens SR, Lokhnygina Y, et al. Outcomes after cardioversion and atrial fibrillation ablation in patients treated with rivaroxaban and warfarin in the ROCKET AF trial. J Am Coll Cardiol. 2013;61:1998-2006.

55. Quinn TJ, Paolucci S, Sunnerhagen KS, et al. Evidencebased stroke rehabilitation: an expanded guidance document from the european stroke organisation (ESO) guidelines for management of ischaemic stroke and transient ischaemic attack 2008. J Rehabil Med. 2009;41:99-111.

56. Rossini R, Musumeci G, Lettieri C, et al. Long-term outcomes in patients undergoing coronary stenting on dual oral antiplatelet treatment requiring oral anticoagulant therapy. Am J Cardiol. 2008;102:1618-23.

57. Ruecker M, Matosevic B, Willeit P, et al. Subtherapeutic warfarin therapy entails an increased bleeding risk after stroke thrombolysis. Neurology. 2012;79:31-8.

58. Southworth MR, Reichman ME, Unger EF. Dabigatran and postmarketing reports of bleeding. $\mathrm{N}$ Engl J Med. 2013;368:1272-4.

59. Spannagl M, Bauersachs R, Debus ES, et al. [Dabigatran therapy - perioperative management and interpretation of coagulation tests]. Hamostaseologie. 2012;32:294-305. 
60. Spyropoulos AC, Douketis JD. How I treat anticoagulated patients undergoing an elective procedure or surgery. Blood. 2012;120:2954-62.

61. Suarez-Pinilla M, Fernandez-Rodriguez A, BenaventeFernandez L, et al. Vitamin K antagonist-associated intracerebral hemorrhage: lessons from a devastating disease in the dawn of the new oral anticoagulants. J Stroke Cerebrovasc Dis. 2013;23:732-42.

62. Valgimigli M, Campo G, Monti M, et al. Short- versus longterm duration of dual-antiplatelet therapy after coronary stenting: a randomized multicenter trial. Circulation. 2012;125:2015-26.

63. Van Ryn J, Stangier J, Haertter S, et al. Dabigatran etexilate-a novel, reversible, oral direct thrombin inhibitor: interpretation of coagulation assays and reversal of anticoagulant activity. Thromb Haemost. 2010;103:1116-27.

64. Wallentin L, Yusuf S, Ezekowitz MD, et al. Efficacy and safety of dabigatran compared with warfarin at different levels of international normalised ratio control for stroke prevention in atrial fibrillation: an analysis of the RE-LY trial. Lancet. 2010;376:975-83.
65. Wanek MR, Horn ET, Elapavaluru S, et al. Safe use of hemodialysis for dabigatran removal before cardiac surgery. Ann Pharmacother. 2012;46:e21.

66. Warkentin TE, Margetts P, Connolly SJ, et al. Recombinant factor VIIa (rFVIIa) and hemodialysis to manage massive dabigatran-associated postcardiac surgery bleeding. Blood. 2012;119:2172-4.

67. Wysokinski WE, Mcbane RD, 2nd. Periprocedural bridging management of anticoagulation. Circulation. 2012;126:486-90.

68. You JJ, Singer DE, Howard PA, et al. Antithrombotic therapy for atrial fibrillation: antithrombotic therapy and prevention of thrombosis, 9th ed. Chest. 2012;141:e531S-75S. 